\title{
Fibrosis and hepatic regeneration mechanism
}

\author{
Esmeralda Zuñiga-Aguilar ${ }^{1}$, Odin Ramírez-Fernández ${ }^{2,3}$ \\ ${ }^{1}$ Universidad Autonoma de Ciudad Juárez, Depto de Ingeniería Eléctrica y Computación, Ciudad Juárez, Chih., México; ${ }^{2}$ Tecnologico Nacional de \\ Mexico, Depto. De Ciencias Basicas, Tlalnepantla de Baz, Mexico; ${ }^{3}$ Facultad de Medicina, HIPAM, Universidad Nacional Autonoma de Mexico, \\ Ciudad de México, Mexico \\ Contributions: (I) Conception and design: Both authors; (II) Administrative support: None; (III) Provision of study materials or patients: None; (IV) \\ Collection and assembly of data: Both authors; (V) Data analysis and interpretation: Both authors; (VI) Manuscript writing: Both authors; (VII) Final \\ approval of manuscript: Both authors. \\ Correspondence to: Odin Ramirez-Fernandez. Instituto Tecnologico de Tlalnepantla, Av. Instituto Tecnológico s/n, La Comunidad, 54070 Tlalnepantla \\ de Baz, Mexico. Email: odinramirezfernandez@gmail.com.
}

\begin{abstract}
Liver cirrhosis is the final stage of continuous hepatic inflammatory activity derived by viral, metabolic or autoimmune origin. In the last years, cirrhosis was considered a unique and static condition; recently was accepted some patients subgroups with different liver injury degrees that coexist under the same diagnosis, with implications about the natural disease history. The liver growth factor (LGF) is a potent in vivo and in vitro mitogenic agent and an inducer of hepatic regeneration (HR) through the hepatocytes DNA synthesis. The clinical implications of the LGF levels in cirrhosis, are not clear and even with having a fundamental role in the liver regeneration processes, the studies suggest that it could be a cirrhosis severity marker, in acute liver failure and in chronic hepatitis. Its role as predictor of mortality in fulminant hepatic insufficiency patients has been suggested. HR is one of the most enigmatic and fascinating biological phenomena. The rapid volume and liver function restoration after a major hepatectomy $(>70 \%)$ or severe hepatocellular damage and its strict regulation of tissue damage response after the cessation, is an exclusive property of the liver. HR is the clinical applications fundament, such as extensive hepatic resections (>70\% of the liver parenchyma), segmental transplantation or living donor transplantation, sequential hepatectomies, isolated portal embolization or associated with in situ hepatic transection, temporary artificial support in acute liver failure and the possible cell therapy clinical applications.
\end{abstract}

Keywords: Fibrosis hepatic; hepatic injury; liver regeneration; fibrosis mechanism; regeneration mechanism

Received: 12 September 2019; Accepted: 10 February 2020; Published: 25 January 2022.

doi: $10.21037 / \operatorname{tgh} .2020 .02 .21$

View this article at: http://dx.doi.org/10.21037/tgh.2020.02.21

\section{Introduction}

The hepatic fibrosis is the common denominator in numerous chronic liver diseases that can progress to fibrosis and hepatocarcinoma. Their frequency and progress are presents in viral hepatitis and non-alcoholic fatty liver; whose prevalence increases in epidemic proportions. Liver biopsy, although imperfect, continues being the gold standard that tends to be replaced in many clinical situations by non-invasive imaging methods.

Hepatic fibrosis is the healing response that occurs as result of acute or chronic liver damage; it is a repair process, dynamic and potentially reversible, that is associated with hepatocellular regeneration. The stellate cells activation causes fibroblasts proliferation and excessive extracellular matrix deposition, which produces fibrous bands and distorts in the liver structure, forming scars and regenerating nodules. As a consequence, liver function is altered and liver blood flow resistance is increased, causing chronic liver disease (CHD) manifested by cirrhosis and complications such as liver failure, portal hypertension and hepatocarcinoma $(1,2)$. 
In industrialized countries the main causes of cirrhosis include hepatitis $\mathrm{C}$ and $\mathrm{B}$ viruses chronic infection, immoderate alcohol consume and nonalcoholic fatty liver disease (NAFLD), which could progress to nonalcoholic steatohepatitis (NASH) and cirrhosis (3). NAFLD has become the most frequent cause of CHD in the United States and Europe, associated with the increase in the prevalence of obesity and the metabolic syndrome. It is estimated that the continuous increase in the prevalence of NAFLD will contribute substantially to the ECH increase and will represent an epidemiological burden in many countries, among which is Mexico (4-6).

The fibrosis diagnosis enhances relevance in recent years due to the fact that several causes could be prevented or treated with the potentially reversible damages, if the causal factor is eliminated $(2,5)$. The clinical management of these patients requires knowing the stage of fibrosis, the content of fat and, possibly, the iron concentration, which frequently coexist, as well as its increase or reduction in the course of treatment.

Liver biopsy has several limitations: it is invasive, expensive, subject to complications, allows to examine only a very small portion of the organ (approximately $1 / 50,000$ of its volume), histological analysis has a great intra- and interobserver variability, with a low efficacy in the initial stages of fibrosis (F1 and F2) and does not provide information about the fibrosis distribution in the parenchyma, which may be heterogeneous 7-9.

\section{Fibrosis evolution}

Fatty liver is a clinical term used by anatomopathologists to describe the condition of the hepatic parenchyma that covers a broad spectrum of disease, ranging from the simple accumulation of triglycerides in hepatocytes (simple steatosis or class 1 ) to the cirrhosis development present in $7-26 \%$ of patients with possible progression to hepatocellular cancer and death, going through steatosis with lobular inflammation (steatohepatitis or class 2), steatosis plus ballooning degeneration (class 3), fibrosis (steatosis plus degeneration ballooning plus Mallory or class 4 bodies) which occurs in $15-50 \%$ of patients, apoptosis, hepatocyte necrosis and obliteration of the hepatic vein (2). Fibrosis can be classified depending on the degree of scar tissue in the tissue according to the following scale:

(I) F0: no fibrosis;

(II) F1: mild fibrosis;

(III) F2: The fibrosis is extended to areas near the portal vein;

(IV) F3: Fibrosis extends out from the portal vein areas. In this stage many fibrosis bridges are connecting the portal vein with the central liver areas;

(V) F4: Fibrosis has evolved to cirrhosis (3).

Usually, during the early disease stages the liver works quite well so that few people experiment symptoms. As the inflammation progresses, the lesions increase and the scar tissue begins to accumulate, the metabolic liver functions begin to alter. If the disease progress, it will inevitably lead to cirrhosis, a state in which the liver is full of scars that interrupt the blood flow and affect the organ functioning. Is normal in the pacients to present symptoms such as fatigue, loss of appetite, vomiting, abdominal pain and swelling, etc. (4).

The development of hepatic fibrosis is a dynamic process that causes the interruption of liver functions with organ dysfunction. Generally, hepatic fibrosis occurs as a response to chronic hepatocellular injury. Many chronic liver diseases are characterized by the deposition of fibrous tissue and the appearance of cirrhosis. During fibrogenesis, type I collagen levels can increase up to 8 times; In addition, the collagen type I/collagen type III ratio also changes, from 1:1 in the healthy liver, to $1: 2$ in the cirrhosis (3). Once this stage is reached, the prognosis is bad, and liver transplantation is the only real alternative to increase survival in these patients. A significant percentage $(2-3 \%)$ of the world population suffers from some type of chronic liver disease, characterized by progressive fibrosis and cirrhosis.

\section{Hepatic injury}

Alcohol is one of the most common causes that generate liver damage, there are many others such as drugs, hepatotropic viruses, nonalcoholic causes, and each of these agents causes liver damage in a different way. In a viral infection, a cascade of mechanism is triggered that eventually leads to the activation of hepatocyte apoptosis mechanism. Any persistent lesion in the hepatic parenchyma can lead to damage, both morphological and functional of the liver, inflammation and necrosis of persistent hepatic tissues are usually described as the cause of fibrosis and nodular regeneration (cirrhosis) which exceeds the capacity of defense and liver repair, leading to the generation of chronic liver damage, which manifests with morphological and functional changes, in the latter case as liver failure (5).

Hepatocytes are approximately $80 \%$ of hepatic volume and non-parenchymal cells for $6.5 \%$ of the total liver, 
in which there are three types of cells: endothelial cells, Kupffer cells (KC) and hepatic stellate cells (HSC) (4).

Most types of hepatic "insult" damage epithelial cells (hepatocytes and/or cholangiocytes), which leads to the release inflammatory mediators and trigger the cascade of antifibrinolytic coagulation. Leukocytes recruited at the site of the lesion phagocytose dead or apoptotic cells and amplify the inflammatory response by generating proinflammatory cytokines, such as tumor necrosis factor (TNF), interleukin 6 (IL-6) and IL-1 $\beta$, and by recruitment of T6 cells. Proinflammatory mediators generated by cellular damage and stimulated immune cells, as well as growth factors and cytokines including platelets derived from growth factor (PDGF), connective tissue growth factor (CTGF), transforming growth factor- $\beta$ (TGF $\beta$ ) and IL-13, activate the mesenchymal precursor cells in the tissues and induce their transdifferentiation to myofibroblasts. Phagocytosis of apoptotic hepatocytes or lymphocytes by hepatic stellate cells also directly triggers their fibrogenic activation. TGF $\beta$ is the main pro-fibrogenic cytokine and positively regulates $\alpha$-smooth muscle actin ( $\alpha \mathrm{SMA}$ ), whereas PDGF induces the proliferation of myofibroblasts through mechanisms regulated by extracellular signal-regulated kinase (ERK) and ERK-independent mechanisms and by changes in intracellular $\mathrm{pH}$. Altered intestinal permeability, particularly in alcoholic liver disease, increases bacterial translocation through the intestinal wall and increases the levels of lipopolysaccharides (LPS) in the circulation, which leads to the activation of hepatic stellate cells and macrophages residing in the liver (also known as Kupffer cells). Toll-like receptor 4 (TLR4) signaling (3).

The process of hepatic fibrosis is not yet fully understood, during the last years the continuous studies about its cellular and molecular biology have allowed to understand many details related to its physiopathogenesis. The identification of the stellate cells of the liver and their great importance in hepatic fibrosis have not only modified the research focus on the pathogenic basis of hepatic fibrosis, but also, they have also opened new lines of interesting research on future therapeutic strategies capable of stopping the fibrogenic process. The HSC are cells located in the Disse space, which in their quiescent state have as their main function serving as a repository of vitamin A and its metabolites. Before aggressive stimuli such as viruses, alcohol or any xenobiotic, through the process of "activation", these cells are transformed to myofibroblasts but with multiple additional functions such as the production of extracellular matrix (ECM) and generation of scar tissue, as well as the expression of actin in its cytoplasm and receptors of PDGF (platelet-derived growth factor) and TGF $\beta$ (transforming growth factor beta) in the cell membrane, produce growth factors, inflammatory cytokines and Fibrogenic [TGF $\beta$, M-CSF (macrophage colonystimulating factor), PAF (platelet-activating factor), IL-10, IL-6], the hepatic myofibroblasts express the tissue inhibitor of metalloproteinase 1 (TIMP1), which inhibits the activity of matrix metalloproteinase (MMP) and increases the accumulation of extracellular matrix (ECM) in scar tissue (5).

The response of the Kupffer cells to a harmful stimulus causes the activation of the HSC inducing the mitotic activity of the same and important additional effects such as: phenotypic transformation of the HSC to myofibroblast with increased synthesis of proteins, elastin and collagen, stimulation of its proliferation, increased response to platelet-derived growth factor and release of mitogenic factors [PDGF, TGF* ${ }^{*}$ IL-1, TNF (tumor necrosis factor alpha), IGF-I (insulin-like growth factor)] that participate in that activation (6).

On the other hand, liver endothelial cells have unions with a certain distance between them called fenestrations, which when a lesion arises becomes a closed capillary to try to protect the hepatocytes and prevent further damage to said cells and release growth factors [bFGF (basic fibroblast growth factor), HGF (hepatocyte growth factor)] and cytokines (IL-6, IL-1) (7).

The activation of HSC constitutes the cornerstone of the pathogenesis in hepatic fibrosis when they differentiate between contractile, proliferative and fibrogenic cells, producing extracellular matrix in excess, in the cirrhotic patient. The key mediators in the activation of stellate liver cells include a series of cytokines, reactive oxygen intermediates and other paracrine and autocrine signals (8).

Significant progress has been made in understanding the molecular aspects of liver cirrhosis. The detailed understanding of this mechanism would be the basis for investigating the therapeutic possibilities in the reversion of fibrogenesis. The cellular and molecular regulation of hepatic fibrosis provides an explanation of the possible reversibility of the process. The accumulation of extracellular matrix in chronic liver diseases is not a unidirectional event, but a dynamic and regulated process that can be intervened (9).

In order to better understand the mechanisms of the disease, different animal models have been used to perform the corresponding studies and analyzes to obtain cirrhotic livers since fibrosis results from the activation of 
cell substances such as cytokines and oxidative stress. The induction of oxidative stress, mitochondrial dysfunction and depletion of antioxidant levels is a relevant feature in the progression of hepatic fibrosis and cirrhosis. According to the dose and administration regimen of thioacetamide (TAA) we can cause acute liver damage or liver cirrhosis. Liver cirrhosis induced by TAA is associated with the exacerbation of lipid peroxidation and the depletion of the antioxidant level. Therefore, the reduction of oxidative stress can be a useful tool to reduce cell damage, cirrhosis and fibrosis in multiple human diseases and in experimental models of hepatic dysfunction (10).

Cirrhosis is the final stage of all chronic progressive liver diseases. It is a diffuse process characterized by the loss of hepatic parenchyma, formation of fibrous septa and regeneration nodules that cause the distortion of the architecture and normal vascular anatomy. Approximately $40-60 \%$ of cases in Europe and North America are due to alcohol and non-alcoholic fatty liver abuse, while $25-30 \%$ is the result of chronic viral hepatitis. Currently, it is considered that cirrhosis is a dynamic and potentially reversible disease in early stages. There are two phases, compensated and decompensated cirrhosis, each with a different prognosis and different survival. Ascites is the most frequent complication, about $60 \%$ of patients with compensated cirrhosis end up developing ascites in the following 10 years (11).

In 1977, experts from the World Health Organization (WHO), based on morphological data, considered the following criteria:

(I) That the process be diffuse to exclude local or focal lesions;

(II) That there is necrosis, which excludes congenital hepatic fibrosis;

(III) There must be nodular regeneration and diffuse fibrosis, excluding regenerative nodular hyperplasia;

(IV) There must be distortion of the architectural pattern and vascular alteration.

These last two criteria are important because they form the basis of diagnosis in biopsies, in the past it was considered that cirrhosis was never reversible, nowadays it is known that, when the fundamental aggression that produced cirrhosis is eliminated, fibrosis could be resolved; this can be observed in patients with hemochromatosis treated with phlebotomies satisfactorily; patients with alcoholic liver disease in alcoholic abstinence; patients with cirrhosis of autoimmune etiology treated with immunosuppressants and chronic hepatitis $\mathrm{C}$ with stage of cirrhosis (F4) with sustained a viral response to antiviral treatment (12-14).

Portal hypertension is one of the most important complications of decompensated cirrhosis and intervenes in the appearance of ascites and hemorrhage due to esophagogastric varices. Hepatocellular dysfunction causes jaundice, coagulation disorders and hypoalbuminemia and contributes to porto-systemic encephalopathy (15-18). Patients who have developed complications from their liver disease and who have decompensated are candidates for a liver transplant. Liver cirrhosis predisposes to the development of hepatocarcinoma.

Initially, fibrosis appears in the periportal and pericentral areas. If the fibrogenesis is perpetuated, bridges or septa of fibrosis are produced that connect the portal spaces and the portal spaces with the centrilobular veins, altering the hepatic architecture and forming nodules of hepatocyte regeneration (19-21). The deposition of extracellular matrix and collagen in the Disse space leads to the formation of pseudomembranes located in the sinusoidal endothelium, which is known as "capillarization of the sinusoids". This produces an additional barrier created between sinusoidal light and hepatocytes that prevents the exchange of substances between sinusoidal blood and parenchymal cells, with hepatocytes being more vulnerable to ischemic and nutritional damage. A process of angiogenesis accompanies the fibrogenic process producing in the liver "neovessels" that may play a role in the pathogenesis of portal hypertension. The destruction of the parenchyma combined with the regeneration and hyperplasia of parenchymal cells, the fibrotic strangulation of the liver tissue and the vascular alterations contribute to the nodular transformation of the liver (22).

\section{Etiology}

Approximately $90 \%$ of the causes of liver cirrhosis in Western countries are alcohol abuse, non-alcoholic fatty liver disease (NASH) and chronic viral hepatitis. Worldwide, chronic hepatitis with hepatitis $\mathrm{B}$ virus $(\mathrm{vHB})$ and $\mathrm{C}(\mathrm{vHC})$ with more than 400 million infected patients represents the most important etiology. The cause of cirrhosis remains unknown in about $10 \%$ of cases (cryptogenic cirrhosis) and approximately $70 \%$ of these cases are believed to be related to NASH in the context of insulin resistance and metabolic syndrome, while the rest may be related to autoimmune mechanisms. Several etiological factors such as hemochromatosis and alcohol, or alcohol and hepatitis C can accelerate progression to cirrhosis (23). 
Infections with $\mathrm{vHB}$ and $\mathrm{vHC}$ are a worldwide public health problem and the most frequent causes of chronic hepatitis, cirrhosis and hepatocarcinoma. Worldwide, it is estimated that around 170 million people have chronic hepatitis due to $\mathrm{vHC}$, and about $20-30 \%$ of patients will have cirrhosis in 20-30 years of evolution.

Longitudinal studies of patients with chronic hepatitis B (HCB) indicate that after diagnosis, the cumulative incidence of developing cirrhosis in 5 years ranges between 8-20\%. In patients with compensated liver cirrhosis, the cumulative incidence of decompensation in 5 years is around $20 \%$, with a probability of survival at 5 years approximately of $80-86 \%$. Patients with decompensated cirrhosis have a poor prognosis, with a probability of survival at 5 years of $14-35 \%$ (24).

Multiple studies have shown the beneficial effects of antiviral treatment with nucleoside/nucleotide analogs, such as tenofovir or entecavir, suppressing vHB replication. The annual incidence of hepatocarcinoma due to HBV in patients with liver cirrhosis ranges from $2-5 \%$ and varies according to the geographical area (25).

\section{Alcoholic cirrhosis}

Excessive and prolonged consumption of alcoholic beverages is one of the main causes of cirrhosis. Likewise, excessive alcohol consumption contributes to a greater progression of liver injury in patients with other liver diseases such as chronic hepatitis due to $\mathrm{vHC}$, hepatic steatosis related to metabolic syndrome, etc. $(26,27)$.

The threshold for developing severe alcoholic liver disease in men is estimated at consumption greater than $60-80 \mathrm{~g}$ of alcohol for 10 years, while women are at risk of developing it by consuming less. The diagnosis of alcoholic liver disease requires a precise interrogation regarding the amount and duration of alcohol consumption. In patients who have had complications of cirrhosis and who continue to drink, 5-year survival is less than $50 \%$. In those who discontinue alcohol consumption and retain abstinence, the prognosis is significantly more favorable; in these, when liver disease is advanced; liver transplantation is a viable option (28).

\section{Non-alcoholic fatty liver disease}

Nowadays the progression of patients with nonalcoholic steatohepatitis to cirrhosis are increasing also the patients with NASH are being identified. Many patients with cryptogenic cirrhosis have NASH.

$\mathrm{NASH}$ is one of the main causes of chronic liver disease in the world, and is closely associated with obesity, diabetes mellitus and metabolic syndromes related to insulin resistance. The progression of the disease to non-alcoholic steatohepatitis with fibrosis and cirrhosis is usually slow and indolent, and in most cases reaches the stage of cirrhosis at the end of life. The estimated rate of development of cirrhosis in about 10 years has been $5-20 \%$ in three studies. These patients have a decompensation rate lower than that of patients with $\mathrm{HCV}$ infection and, like them, can develop hepatocellular carcinoma (29-32).

\section{Autoimmune liver cirrhosis}

Many patients with autoimmune hepatitis have established cirrhosis at the diagnosis of liver disease. Autoimmune hepatitis has been able to have an asymptomatic and indolent course or to begin with some complication of decompensation of cirrhosis. The diagnosis requires the exclusion of other causes of liver disease; antinuclear autoantibodies (ANA), smooth anti-muscle (SMA), hepatorenal antimicrosomal (anti-LKM-1) or soluble antiantigen hepatic (antiSLA) and hypergammaglobulinemia are usually present. Immunosuppressive treatment with prednisone, azathioprine or with the combination of both drugs improves prognosis and may slow liver fibrosis and prevent complications of the disease (33).

\section{Primary biliary cirrhosis (PBC)}

PBC has a prevalence of around 100-200 patients per million inhabitants, and is more frequent in middle-aged women. The presence of antimitochondrial antibodies (AMA) antiM2 diagnoses the disease in almost $90 \%$ of patients. Ursodeoxycholic acid is the only approved treatment that has a certain degree of efficacy and that reduces the progression of the disease. Liver transplantation is the indicated treatment in patients with decompensated cirrhosis (34).

\section{Biliary cirrhosis secondary}

Biliary cirrhosis is the result of necroinflammatory lesions, congenital or metabolic processes or extrinsic understanding of the bile ducts that produce a chronic cholestasis syndrome and, consequently, prolonged interruption of bile flow, as occurs in primary sclerosing cholangitis, idiopathic 
ductopenia of the adult, cystic fibrosis, etc. Two categories reflect the anatomical sites of the interruption of the bile flow: intrahepatic and extrahepatic. The manifestations of terminal liver disease in this type of cirrhosis are the same as in those of another etiology (34).

\section{Heart cirrhosis}

Patients with chronic congestive heart failure on the right side develop chronic liver damage and cardiac cirrhosis. This is an increasingly rare cause of liver cirrhosis (35).

\section{Hemochromatosis}

Hemochromatosis is a hereditary disorder of iron metabolism that produces a progressive increase of iron in the liver that causes hepatic fibrogenesis and which progresses to cirrhosis and the development of hepatocarcinoma. The prevalence of hemochromatosis is high, with a genetic susceptibility that occurs in one of every 250 individuals. The diagnosis is established by the determination of iron metabolism parameters, mainly the transferrin saturation index and ferritin concentration, which will be very high. The study of HFE gene mutations also plays a relevant diagnostic role. The treatment is carried out with therapeutic phlebotomies that are performed regularly (36).

\section{Wilson's disease}

There are other less frequent causes of chronic liver disease that can progress to cirrhosis as metabolic-hereditary liver diseases such as Wilson's disease; cystic fibrosis and deficit of $\alpha 1$ antitrypsin affecting young patients. The assessment of the levels of ceruloplasmin, cupremia and cupruria suggests the diagnosis, which if necessary can be confirmed with the quantification of copper in the liver biopsy. The presence of the Kayser-Fleischer ring can be of great diagnostic help (37).

\section{Pathogeny}

The following physiopathological mechanisms are important in the development of hepatic cirrhosis of any etiology:

(I) Necrosis or lysis of hepatocytes with loss of hepatic parenchyma and inflammation;

(II) Fibrogenesis (deposit of extracellular matrix);

(III) Changes in cell growth (hyperplasia, regeneration);
(IV) Vascular and circulatory alterations.

The necrosis and lysis of hepatocytes and chronic and continuous inflammation constitute a stimuli and perpetuating factor of proliferation and growth of the hepatocytes and of the process of fibrogenesis. In cirrhosis, pro-fibrogenic cytokines such as transforming growth factor $\beta 1$ (TGF- $\beta 1$ ) are produced that initiate and perpetuate the activation of transformed hepatic stellate cells in the myofibroblast phenotype (14). Myofibroblasts contract, proliferate and produce collagen and other components of the extracellular matrix. The development of cirrhosis is accompanied by a marked increase in collagen content and deposition of extracellular matrix produced mainly by activated star cells transformed into myofibroblasts (38).

\section{Compensated cirrbosis}

The clinical course of compensated cirrhosis is not known defined due to the asymptomatic or oligosymptomatic character of this phase. The mortality prognostic factors that are identified with a higher frequency in this compensated phase are related to the presence of portal hypertension (platelet count, spleen size or presence of varicose veins). Probably, these prognostic factors identify patients with greater risk of developing complications characteristic of the decompensated phase of the disease (39).

\section{Decompensated cirrbosis}

Decompensated cirrhosis is defined by the presence of ascites, variceal hemorrhage, hepatic encephalopathy and/ or the appearance of jaundice. Ascites is the complication that marks the beginning of the phase of decompensated cirrhosis in most cases; therefore, it is considered the key sign of decompensated cirrhosis. Once this phase is reached, the prognosis with respect to survival markedly worsens with 1 -year survival and at 2 years of $61 \%$ and $50 \%$, respectively, and a 10-year survival rate of $7 \%$ (40).

The identification of prognostic factors of mortality in the decompensated phase is of great relevance, since these patients are the ones with the highest risk of death $(41,42)$. The factors that are most frequently identified are those associated with circulatory dysfunction with impaired renal function, the presence of hepatocellular carcinoma and the variables associated with greater deterioration of liver function. Four clinical stages or "status" of cirrhosis with a different prognosis can be identified: 
(I) Stadium 1. It is characterized by the absence of esophageal varices and ascites. While patients remain in this stage, the mortality rate is less than $1 \%$ per year. Patients leave this stage with an accumulated rate of $11.4 \%$ per year, $7 \%$ due to the development of varicose veins and $4.4 \%$ due to the development of ascites (with or without varicose veins);

(II) Stadium 2. It is characterized by the presence of esophageal varices without ascites and without bleeding. While patients remain in this stage, the mortality rate is $3.4 \%$ per year. Patients go to another stage to develop ascites (6.6\% per year) or to present variceal hemorrhage before or at the same time as the development of ascites (rate 4\% per year);

(III) Stage 3. It is characterized by the presence of ascites with or without esophageal varices in a patient who has never bled. While patients are in this stage, the mortality rate is $20 \%$ per year, significantly higher than in the other stages. Patients usually leave this stage due to variceal hemorrhage ( $7.6 \%$ per year);

(IV) Stadium 4. It is characterized by digestive hemorrhage by varicose veins with or without ascites. At this stage, the annual mortality rate is $57 \%$ (about half of these deaths occur in the 6 weeks after the initial episode of digestive bleeding).

Stages 1 and 2 correspond to patients with compensated cirrhosis, while stages 3 and 4 refer to decompensated cirrhosis. Hepatocarcinoma can occur at any stage of cirrhosis with a constant rate of $3 \%$ per year (42).

Liver transplantation significantly improves the survival and quality of life of patients with end-stage cirrhosis. However, a significant proportion of patients die in the transplant waiting list, due to the insufficient number of donors. The adequate prediction of life expectancy in these patients is very important.

\section{Anatomopathological vision}

A healthy liver has a small amount of collagen and connective tissue. When a chronic insult occurs that produces a continuous hepatic lesion, a dynamic process of continuous formation, degradation and remodeling of the extracellular matrix occurs, resulting in a progressive accumulation of extracellular matrix. Likewise, the connective tissue can be degraded and fibrosis can return to establish different specific treatments in chronic liver diseases $(43,44)$.

\section{Hemodynamic vision}

Portal hypertension develops progressively in the natural course of chronic liver disease and is the main prognostic factor of it. It is defined by an increase in the hepatic venous pressure gradient (GPVH) above normal values $(1-5 \mathrm{mmHg})$ and is considered clinically significant above $10 \mathrm{mmHg}(24,25)$, a value from which the complications of portal hypertension and the appearance of esophagogastric varices. Gastrointestinal hemorrhage and ascites occur when the GPVH is above $12 \mathrm{mmHg}$. In decompensated cirrhosis, a GPVH above $20 \mathrm{mmHg}$ is an important predictor of poor prognosis of variceal hemorrhage and the development of refractory ascites, hyponatremia and hepatorenal syndrome $(44,45)$.

\section{Prognostic indexes}

Adequate assessment of the prognosis of life in patients with cirrhosis is of great clinical relevance, in that it contributes to making management decisions in different clinical scenarios such as the indication for surgery, transjugular intrahepatic portosystemic shunt (TIPS) or liver transplantation. In the last fifty years, various clinical tools have been developed for this purpose. One of the best known and used is the Child-Pugh scale, designed in 1964 by Child and Turcotte and later modified by Pugh. This scale was designed to evaluate the mortality associated with transection surgery of esophageal varices, but with time it was extended to the evaluation of mortality of cirrhotic patients at 1-2 years, without surgery. This classification has limitations, such as the inclusion of two variables whose evaluation can be subjective, so a decade ago, researchers from the Mayo Clinic in the United States developed the MELD score system (model end-stage liver disease) (46).

The Child-Pugh score (5-15 points) is the result of the sum of the score of each of the 5 variables. In this way, it is determined: Child A: 5-6 points; $0 \%$ mortality at 1 year and $15 \%$ at 2 years; Child B: $7-9$ points; $20 \%$ mortality at 1 year and $40 \%$ at 2 years; Child C: $10-15$ points; mortality $55 \%$ at 1 year and $65 \%$ at 2 years.

The characteristics of this index and its wide use in the allocation of organs for liver transplantation have determined that it is now considered the best tool to estimate the shortterm prognosis of patients with cirrhosis 29-31 (47).

The MELD system was developed from the analysis of 231 cirrhotic patients who underwent the placement of a 
TIPS. Through a rigorous statistical analysis, a formula was derived to predict the mortality associated with the intervention based on 3 objective variables: bilirubin $(\mathrm{mg} / \mathrm{dL})$, creatinine $(\mathrm{mg} / \mathrm{dL})$ and prothrombin time (INR). The MELD score correlated with the mortality observed at 3 months in these patients. This index is applied today in many liver transplant units $(47,48)$.

The MELD index has been validated in various publications of cirrhotic patients of different etiologies and with different degree of severity. All the studies have shown that the MELD index is reproducible and with an excellent predictive capacity of the mortality of ambulatory and hospitalized cirrhotic patients, both at 3 months and at 1 year. For this reason, it was recommended that patients with a MELD score greater than 1,529 be candidates for transplantation. An exception to the prioritization by MELD score is patients who present hepatocarcinoma with an indication for liver transplantation.

\section{Steatosis during regeneration}

As it has been verified in numerous experiments, during the initial processes of the hepatic regeneration (HR), it is normal that the hepatocytes accumulate drops of fat in his interior of temporary form to the 24 to 72 hours after a partial hepatectomy, and tends to disappear by itself; same Possibly this occurs as a hepatocellular metabolic response to damage, to generate energy sources and material for the construction of membranes; however, the mechanism with which the liver manages to do so remains to be elucidated $(48,49)$.

It has been proposed that liver regeneration is due to a process of cell regeneration mediated by four waves of replication: one that exhibits the greatest magnitude, two of medium intensity and one of last, which seem to follow circadian rhythms, showing mitotic picks and they are related to three waves of liver fat accumulation, but the mechanism involved is still not known.

Multiple studies have been conducted to try to check the mechanism of fat accumulation without major results, however, recent studies have been able to clarify that the accumulation of fats during liver regeneration would not necessarily depend on de novo synthesis in the liver of acids fatty acids, neither of the reabsorption of these at intestinal level but of a transport from adipose tissue to the liver (49).

\section{Role of peripheral fatty tissue}

The steatosis that occurs physiologically during liver regeneration is that the fat comes from the peripheral fatty tissue. For this, mice with lipodystrophies were evaluated, in these studies new evidence is shown that disruptions in glucose metabolism can alter HR, it is thought that hypoglycemia consequent to a hepatectomy could cause a lipolysis in adipose tissue that would contribute to fatty deposits in the liver and, therefore, regeneration, also showing reduced levels of adiponectin in diseased mice, consistent with a history in which adiponectin levels are related to liver regeneration (50).

The first mechanism, an increase in the arrival of fats, could cause a depletion of adipocytes, which would be unable to capture circulating energy metabolites. Insulin is an important factor that removes triglycerides from lipoproteins, being an important factor in hepatocyte fatty metabolism, possibly these depleted adipocytes cause an insulin resistance to protect them from a surcharge and prevent tissue damage and cellular. The adipocytes could overflow fat to the surrounding tissue such as muscles and liver (50).

When the lipid reserve capacities of the liver, after the overflow of the adipocytes, it is exceeded, it releases fatty acids into the bloodstream, accumulating in organs and in the walls of the vessels. The macrophages, to try to buffer the present lipids, phagocytize and metabolize them, transforming themselves into foamy cells and releasing, as waste of the metabolism of phagocytosed lipids and releasing EROS (51,52).

In the second case (an increase in lipid intake), the lipids must be transported by lipoproteins from adipose tissue to the liver. Once inside the hepatocytes, these require a series of intracellular transport proteins such as FATP or FAT/ CD36, FABP, caveolins-1 among others, therefore lipid accumulation in hepatocytes requires a great interaction between multiple proteins. This suggest that a deficiency or overexpression in any of these proteins could lead to alterations in the lipid metabolism of hepatocytes. In the third case with respect to a decrease in lipid output, when the intake and synthesis of lipids increases in the liver, this in turn is responsible for increasing the output or excretion of these.

The regulation of triglyceride synthesis in the liver depends on insulin signaling and the activation of the transcription factor SREBP-1c that modulates lipid synthesis. A genetic change that induces insulin bypass by the SREBP-1c gene could cause an increase in triglycerides without increasing cholesterol. In the specific case of alcohol, it stimulates the activation of SREBP through 
PPAR signals. Obesity and insulin resistance, being related to inflammatory processes and cytokine signaling that produce an overexpression of SREBP. The concentration of bile acids, whose increase is translated into an increase in SREBP, is also involved in hepatic steatosis (50-52).

The second hit has been associated with immunological reactions such as the complement system, which would be involved in the pathogenesis of multiple diseases such as alcoholic fatty liver disease and viral hepatitis. It has been shown that complement activation is associated with NAFLD due to the damage caused by the activation of the membrane attack complex, which would be related to the accumulation of neutrophils and the increase of apoptosis in diseased livers.

High concentrations of neutrophils could be associated with the secretion of proinflammatory cytokines such as $\mathrm{TNF} \alpha$, produced during inflammatory responses associated with obesity and that could produce the accumulation of fats, TGF $\beta$, involved in the processes of fibrosis mediated by stellate cells of the liver, IL-6, which sensitizes the liver to apoptosis, IL-10, whose deficiency could be related to the progression of the disease, among other cytokines10 (53-55).

Another possible explanation for the second hit is an alteration in adipokines, specifically visfatin, which is a signal synthesized by white adipose tissue that along with other proinflammatory signals such as IL-6, TNF- $\alpha$ and IL-8 could be related to NAFLD $(56,57)$.

\section{Risk factors of NAFLD}

The pathogenesis of NAFLD is related to diabetes mellitus type 2, making studies of screening for this disease could serve to perform an early diagnosis and prevent the development of complications, such as cardiovascular diseases. The presence of hyperglycemia in the blood is usually evaluated. The sensitivity of this test depends on the population evaluated. However, it has not been established which patients with NAFLD may have an oral glucose intolerance (58).

Approximately $48.7 \%$ of the patients with non-alcoholic fatty liver disease had some type of metabolic disorder such as Type 2 diabetes mellitus. This group had a higher age, higher body mass indexes and lower HDL-C12 indexes.

It is shown that there is a relationship between glucose deregulation and NAFLD. Age is an important factor in the development of NAFLD along with high body mass indexes. It is observed that patients with NAFLD have low levels of HDLD-C, lower albumin serum levels, which may be related to an advanced stage of liver disease which may determine that metabolic disorders, age and mass index are important risk factors for the development of a nonalcoholic hepatic steatosis $(58,59)$.

\section{Clinical manifestations of NAFLD}

Hepatic steatosis is a clinical picture that is usually asymptomatic, detectable in the physical examination if there is any type of hepatomegaly. It can usually be evaluated by observing an increase in aminotransferases once a chronic liver disease has been ruled out. The radius of values of aspartate aminotransferase and alanine aminotransferase is lower in the presence of steatosis, but its predictive value is poor in patients with severe steatosis and parenchymal fibrosis (59). Serum levels of alkaline phosphatases and gamma-glutamyl transferase are generally above normal levels, with serum lipid levels and glucose concentrations also being elevated in about $75 \%$ of patients (60). Other possible laboratory abnormalities are hypoalbuminemia, prolonged prothrombin time and hyperbilirubinemia, but these are strange findings in patients with advanced disease. Other good mechanisms to evaluate it are the waist radius and the body mass index (BMI), which have been shown to be good indicators for hepatic steatosis.

There are multiple theories that could explain it. One of them is that the fibrosis produced by the advanced state of NAFLD due to the increase of oxidative stress would reduce the capacities of mature hepatocytes to proliferate, which would produce a dispare HR $(59,60)$.

Other theories explain these processes, pointing out that leptin, for example, is an important factor in the murine models of hepatic steatosis, since, to induce it, they are used either mice with their delegate or knock-out gene or models with rich in fat diets.

A possible detonating factor that could affect HR during steatosis processes due to its role in lipid metabolism is fatty acid synthase. It has been shown that its overexpression is related to cancer and in multiple neoplastic diseases, while its suppression is related to the decrease in cell survival, however, an inhibition of the expression of this, does not alter the accumulation of fats in the liver during the regeneration periods, which could suggest that its role would be rather secondary (60).

The reticulum (ER) is one of the cellular organelles responsible for the synthesis, folding of proteins and the movement of intracellular calcium, an increase in 
stress of ER, is associated with various chronic diseases. Studies in livers with steatosis demonstrated the ERs of fatty hepatocytes had a lower response to stress, added to the fact that the low expression of chaperone proteins responsible for protein movement within the organelle and the decreased expression of proteins responsible for initiating signaling proapoptotic could explain the low rates of apoptosis in fat cells $(60,61)$.

Possibly, this protein is responsible for disinhibiting the cyclin/CDK pathway, through the inhibition of factor CDKN1A which is an important factor to get out of the processes of differentiation and proliferation in the liver. It also proved to be a potential inhibitor of the leptin pathways, without inhibiting caveolins or glucocorticoid receptors, thus providing new evidence that the leptin pathways are not directly involved in regeneration processes (61).

\section{Function of Kupffer cells}

Kupffer cells are macrocytic cells of the liver. There are several theories that involve these cells to explain the disparate regeneration produced by steatosis. One of them implies that the accumulation of fatty acids inside the liver cells promotes an increase in the reactive oxygen species increasing the oxidative stress of the mitochondria and lipid peroxidation. Added to these livers with steatosis have a poor elimination of free radicals which cause the accumulation and activation of Kupffer cells, starting to produce proinflammatory cytokines such as $\mathrm{TNF}-\alpha$ and IL-6 that could increase liver damage $(50,62)$.

Kupffer cells recognize pathogen-associated patterns or patterns presented by damaged cells through TLR receptors whose regulatory proteins (CD14 and TLR4) lead to sensitization towards saturated fatty acids which can activate the TLR4-mediated and continue with inflammation, although they also recognize some cytokines, such as PPAR $\alpha$, which is necessary for liver regeneration to occur and is associated with the accumulation of fats.

Previous studies have shown that livers with a mild steatosis without inflammatory response do not present a difference in the liver regeneration of healthy livers, something that did not happen with livers with severe steatosis, and there was an increase in the proinflammatory cytokines produced by Kupffer cells $(50,56,61)$.

This poses to the Kupffer cells explain 4 possible mechanisms in which these cells may be involved in the regeneration and liver damage by steatosis:

(I) The cell growth of the hepatocytes produces an odd perfusion; the leukocytes are trapped in these reduced spaces which can activate the leukocytes;

(II) The constant interaction of Kupffer cells with fatty acids can activate proinflammatory pathways and resistance in insulin through contact with the surface and activation of extracellular receptors and intracellular mediators;

(III) The lipids of the membrane are changed and can cause response of Kupffer cells;

(IV) The abundance or abnormality of lipids in the membrane could cause that the cells of Kuppfer do not recognize the hepatocytes.

Although there have been multiple studies in different genetic and biochemical models, to analyze the effects of hepatic steatosis on regeneration and metabolism, it is not yet possible to determine an exact path that effectively alters the normal physiological processes of this organ (63).

Several models have been proposed that relate the etiology of NAFLD to biochemical, genetic or inflammatory processes, but which are not yet possible to integrate correctly into a single large model that explains it and that can demonstrate how regeneration can affect, which could It is very useful for the clinical practice and treatment of this syndrome that is becoming more common in the world (64).

\section{Animal models}

Regeneration has been studied in multiple experimental models; from cell cultures, to "in vivo" models of hepatopathy induced by toxins (Cl4C, galactosamine, thioacetamide), bacterial products (LPS), viruses, and in surgical models such as $70 \%$ hepatectomy, or partial liver transplantation. It has also been studied in genetically modified animals in which a gene has been overexpressed (knock-in) or in knockout animals (KO) in which the specific or conditional deletion of a gene has been induced (62). Other authors have carried out transcriptional studies analyzing the expression of thousands of genes, trying to obtain a representative pattern of regeneration. Although $\mathrm{KO}$ models have been crucial to identify the signaling and transcription pathways of the $\mathrm{RH}$, due to the pleiomorphic and redundant character of the regeneration, its interpretation is difficult.

The most commonly used model is $70 \%$ hepatectomy in rodents, since it represents the greatest stimulus for regeneration and guarantees the synchronous and homogeneous response of the remaining liver. This model has allowed to compare regeneration in control animals- 
"wild type" - with genetically modified animals for signaling molecules, their receptors and cell cycle regulatory factors. After a hepatectomy or an acute injury, the recovery of the hepatic mass is due to a "compensatory hyperplasia" of the residual liver, and not to an epimorphic regeneration of the lost tissue, as occurs in lower vertebrates-Zebra or Salamander-which the capacity to regenerate the amputated limbs or other structures-the tail, the lower jaw, the cardiac ventricle-throughout his life (62-64).

The $\mathrm{RH}$ begins with the recognition of the molecular patterns associated with pathogenic microorganisms (PAMP) or of the molecular patterns associated with cell damage (DAMP). Generating an innate immune response-activation of complement $\mathrm{C} 3 \mathrm{a}$ and $\mathrm{C} 5 \mathrm{a}$ fractions, production of TNF-a, synthesis of IL-6, IL-1b, IL-18-that stimulate the division of hepatocytes through the transition of the basal state G0 to the first phase G1 of the cell cycle (phase G1, of the English Gap) (preparation phase) and that occurs in the first 4 hours after a hepatectomy. the RH, the phases of the cell cycle are reproduced which has aroused great interest in knowing the gene expression (65). Cell proliferation occurs in a chronological and sequential order; first the hepatocytes are divided and later the Kupffer and endothelial cells, followed by the neoformation of vessels and bile canaliculi, until the liver structure is reproduced. After a hepatectomy of $70 \%$; $95 \%$ of the hepatocytes pass in 4 hours from the G0 phase to the G1 phase. Alterations in the regulation of the cycle 192, 195 TNF-a Stimulus for the transition G0 to G1 of the cycle 44, 72, $118 \mathrm{Gp}$ 130 Alteration in the RH after the administration of LPS 72, 196 IKK2 Deletion of the inhibitor IKK2. Induces the innate response and proliferation of hepatocytes 196 Complement $\mathrm{C} 3 \mathrm{a} / \mathrm{C} 5$ a Complement factors are crucial in the initial phases of the RH 170, 199 Farnesoid X receptor (FXR) FXR-deficient mice suffer a delay in HR and increase in mortality proliferation phase C-met HGF and MET receptor are essential at the start of the cycle after HP FosM1b Overexpression stimulates entry into hepatocyte mitosis EGF Increase mortality after HP $(65,66)$. Delay in the division of hepatocytes in mice K ILK inhibitor KO mice increased proliferation and hepatomegaly TGF-a Overexpression develops hepatomegaly. Increase of DNA synthesis phase cessation and regulation TGF-b Transgenic animals suffer delay in cell proliferation TGF-b R2 KO mice show early DNA synthesis, KO mice develop high degree of ploidy. p53 regulates the three phases of the $\mathrm{RH}$ at the end of which there is the critical point of control, called a restriction (" $R$ "), which determines whether the cells are irreversibly divided. Until then the process is reversible and the hepatocytes could return to their previous state (G0) if the ideal conditions (growth factors, cytokines, etc.) were not given. Subsequently, they progress through the phases of the cell cycle to the cell division or cytokinesis of the hepatocytes.

At 30 minutes after a hepatectomy, an increase of the preformed transcription factors in the cytosol has been described in response to the binding of cytokines to their receptors: the signal transducer and transcription activator STAT3 (activator of transcription and transducer signal 3), nuclear factor Kappa-b (NF-Kb) and activating protein 1 (AP-1); that stimulate the synthesis of proteins and the expression of the genes necessary to initiate the cell cycle (preparation phase). In the rat, an increase in DNA synthesis (S phase) has been described 24 hours after the hepatectomy, and in the mouse at 36 hours. Hepatocytes enter mitosis synchronously at 48 hours, followed by Kupffer cells. Stellate cells and those of the biliary epithelium have their $\mathrm{S}$ phase at 48 hours and proliferate more slowly (67). Endothelial cells initiate their proliferation at 3 days, expressing a peak at 5 days after hepatectomy. At 72 hours after hepatectomy, a subpopulation of the hepatocytes returns to basal state G0, while others reinitiate mitosis until their return to the previous state G0, with the restoration of the hepatic mass (68). It has been postulated that this second proliferative peak is due to growth factors synthesized by the hepatocytes themselves or to co-mitogens such as norepinephrine, insulin, somatostatin and glucagon. From the morphological point of view, accumulations of hepatocytes are observed in the first hours (mononuclear and binucleated cells with different degree of ploidy: tetraploid $-4 n-$, octaploid $-8 n-;-16 n-,-32 n-)$. At 2-4 days (69-71).

The correlation with the phases of the hepatocyte cell cycle is shown on the abscissa axis. Phase $\mathrm{S}$ Mitosis Cytokinesis Day 1 Day 2 Day 3 Day 4 Day 5 Day 6 Day 7 Day 8 Day 9 Day 10 Hepatocytes C. Kupffer Colangiocytes C. Sinusoids 6h 12h 18h 24h 30h 36h G1 G2 G1 G2. The proliferation of stellate and sinusoidal cells is observed, increasing the size of the lobes $(72,73)$.

The relative hypoxia of the remaining liver induces the expression of transcription factor, induced by hypoxia (HIF-1a)—after 12-48 hours of partial hepatectomy-that activates the endothelial-vascular growth factor (VEGF), the fibroblast growth (FGF) and inducible nitric oxide synthetase (iNOS) (74-77). The proliferation of hepatocytes progresses from the periportal areas to the pericentral areas of the lobule. Once the initial volume is re-established, 
there is a phenomenon of apoptosis, tissue remodeling and zonalization of the hepatocytes.

Unlike other solid organs such as skin or intestine, $\mathrm{RH}$ is due to the proliferation of mature hepatocytes and mesenchymal cells: Kupffer cells, endothelial cells, stellate cells, NK cells, and not proliferation and differentiation of cells. Pluripotent stem cells or oval cells; although its proliferation has been described when HR is insufficient or is abolished, as in chronic liver diseases. With the identification of oval cells-precursors of hepatocytes and cholangiocytes-, as well as the discovery of the tissue plasticity of hematopoietic stem cells, endothelial progenitor cells (CD133, CD117) and induced pluripotent stem cells (iPS), In spite of the enormous expectations, the results have been scarce even in the trials carried out with isolated hepatocytes in the congenital errors of the metabolism, being limited in the majority of the cases to a temporary improvement (78).

Recently, preliminary studies have been published by the infusion of bone marrow growth factors (granulocyte stimulating factor, G-CSF) in patients with the acute-onchronic syndrome, and with the infusion of bone marrow progenitor cells (CD133). The regenerative capacity of the liver is almost unlimited. Up to 7 consecutive $50 \%$ hepatectomies have been described in the rat, without causing liver failure or diminishing its regenerative potential (2-4). In a model of homozygous tyrosinemia in the mouse $\left(\mathrm{FAH}^{-/-}\right.$Miles), hepatocyte transplantation corrected the metabolic deficit. When the transplanted hepatocytes were isolated, they reversed the enzyme deficit in a second generation of $\mathrm{FAH}^{-/-}$mice; and so on up to ten generations! It has been estimated that the hepatocytes of a mouse could suffer up to 69 duplications (generate 50 mouse livers) $(79,80)$.

Another enigma of the $\mathrm{RH}$ is the strict regulation between the hepatic volume and the body surface, expressed as liver index (liver weight/total weight $\times 100 \sim$ $2.5 \%)$, known as "hepatostatic" regulation. In the clinical and experimental transplantation-small grafts in large recipients and vice versa - the adaptation of the donor liver volume to the recipient's body surface has been confirmed. This control has been described even in the baboon xenotransplantation of man. Although regeneration has been linked to changes in the nucleus, cell division must duplicate the cytoplasmic organelles. Most of the growth factors [PDGF, epidermal growth factor (EGF), hepatocyte growth factor (HGF) and insulin growth factor (IGF-1)], besides having mitogenic effects, increase the size and cell survival, inhibiting apoptosis. In the lower vertebrates and mammals, polyploid, tetraploid (4n), octaploid (8n), either mononucleate or binucleated hepatocytes $(2 \times 2 n ; 2 \times 4 n ; 2$ $\times 8 \mathrm{n}$ ) are frequent due to an alteration in cytokinesis. The degree of ploidy varies according to the species; in rats, $80 \%$ of hepatocytes are polyploid and aneuploid, $60 \%$ in the mouse and $30-70 \%$ in the human being (80).

The first consequence of ploidy is the increase in the size of hepatocytes. It has been postulated that the cell size depends on the nuclear content of DNA, so that a larger number of chromosomes allows to increase the size. After a hepatectomy in rodents, a decrease in binucleated hepatocytes from $20 \%$ to $5 \%$ and an increase in tetraploid hepatocytes $-4 n$ - and octaploids $-8 n$ - have been described; and even hepatocytes $16 \mathrm{n}$ at 72 hours, expressing that hepatectomy is a very intense stimulus for DNA duplication prior to cytokinesis.

In animals in which chromosome separation was inhibited, the recovery of the hepatic mass after a hepatectomy was due to the polyploidy of the higher hepatocytes $-18 \mathrm{n}, 32 \mathrm{n}$ ). This phenomenon-known as endoreduplication-gives rise to multiple replicative sequences of the chromosomes without cell division, generating cells with several copies of the genome, capable of increasing gene expression $(80,81)$. It is striking that in these cases the liver function was normal, confirming the functional equivalence between hepatocytes $2 \mathrm{n}, 4 \mathrm{n}$ and $8 \mathrm{n}$. Now we got a model that described the regulatory role of $\mathrm{p} 53$ in the integrity of the genome, both in quiescent hepatocytes and during regeneration (7 days posthepatectomy). These authors have described that $\mathrm{KO}$ mice for $\mathrm{p} 53\left(\mathrm{p} 53^{--}\right)$express a greater degree of ploidy than $\mathrm{p} 53^{+/+}$control mice, and that they initiate division before, develop a larger size of the nucleus and the cytoplasm, and a degree greater of ploidy than $\mathrm{p} 53^{+/+}$mice (wild type or control) after a hepatectomy of $70 \%$.

The absence of $\mathrm{p} 53$ alters the reversion of the ploidy, so that regeneration in $\mathrm{p} 53^{-/-}$animals is primarily due to the increase in cell size. Although recovery of the hepatic mass at 7 days after hepatectomy was equivalent in both groups of mice, these authors observed more errors in chromosomal integrity in $\mathrm{p} 53^{-/-}$mice (multipolar spindles and lazy chromosomes) than in control animals $\left(\mathrm{p} 53^{+/+}\right)$. Several authors have highlighted that the more severe the liver damage (extreme resections $>70 \%$ of the parenchyma, transplantation between alive with grafts $\leq 30 \%$, submassive hepatic necrosis...), the more intense is the proliferative response of the liver, and vice versa. 
The liver-despite its metabolic complexity-maintains homeostatic functions: protein synthesis, coagulation factors, anti-proteases, detoxification of xenobiotics, etc. during regeneration. Studies with microarrays have shown a selective response in the first 40 hours, prioritizing the expression of genes related to cell division and silencing those related to hydrocarbon metabolism. Studies with optical and electronic microscopy have revealed, at 24 hours of a hepatectomy of $70 \%$, selective changes in the hepatocytes of zone 1, in contrast with zone 3 of the hepatic lobule, in this work the authors reported that there are three peaks in the DNA synthesis of hepatocytes, initially in zone 1 and later in the intermediate zone of the lobule. Curiously, $15 \%$ of the hepatocytes do not divide, while $11 \%$ of the hepatocytes divide at least three times, the cause being unknown (82).

The liver is, with the brain, the organ with the greatest capacity to maintain its integrity -morphological and functional- and respond to the most varied alterations of the internal environment (82). This property is present in other "biological systems": physiological homeostasis, tissue development, cell cycle, ecological resistance, circadian rhythm and even cancer. This property has been called "robustness" or "strength" and has aroused great interest in evolutionary biology during the last decade. HR meets the required qualities: pleiomorphism, redundancy and biofeedback mechanisms. This makes their understanding difficult and that, still, it has not been possible to intervene in regeneration for therapeutic purpose). The $\mathrm{RH}$ can be divided into three phases: an initial phase of "preparation" or "priming", which corresponds to the passage of the quiescent hepatocytes from the G0 phase to the G1 phase of the cell cycle, which occurs in the first 4 hours after the hepatectomy. A second phase, or "progression" phase, corresponding to the transition from the G1 phase to complete mitosis (cytokinesis); and a third phase of apoptosis, tissue remodeling and return to phase G0 (83-85). The model described has been confirmed in different species and has the virtue of corresponding with the phases of the cell cycle and of which some basic aspects are described. The cell cycle is divided into four phases: G1, S, G2 and M. DNA synthesis, and duplication of chromosomes, occurs in the $\mathrm{S}$ phase (synthesis). The segregation of the chromosomes takes place in the $M$ phase or mitosis, which is divided into four stages: prophase, metaphase, anaphase and telophase. Cell division ends with cytokinesis, giving rise to two similar cells that can restart the cycle or return to the resting state G0. The phases G1 and G2 (from the English "Gap") or "spaced" or "preparative" phases located before the S phase (DNA synthesis) and the $M$ phase (mitosis) respectively, provide time for cell growth and regulate the transition to the next phase depending on intracellular and extracellular signals (86).

In the cycle there are three control points: the "R" or restriction point that regulates the entry in the cycle at the end of phase G1; and the two control points of mitosis; the G2/M point that controls the start of mitosis and the control point of the metaphase-anaphase transition that results in the segregation of the "sister" chromatids and at the end of mitosis. The " $R$ " point regulates the speed of cell division and determines the irreversibility of the cycle. Once this point has been passed, the cell will inexorably divide, although it will continue to be subject to the control mechanisms. The majority of mitogens and growth factors-platelet-derived growth factor (PDEGF), EGF, HGF and transforming growth factor b (TGFb)-, exert their action at the " $\mathrm{R}$ " checkpoint when the cell is more sensitive to exogenous factors (87).

The necrosis induces the response of the innate immune system due to the recognition of the DAMP by the recognition receptors of said patterns (RRP). These receptors have been described in the plasma membrane, in the endosomal membranes and in the cytoplasm of mesenchymal cells and hepatocytes. Depending on the severity of the lesion and the regenerative response, the lesion will be reversible or irreversible. In the latter case the liver is unable to restore homeostasis, which is associated with a very high mortality and whose only alternative is liver transplantation (fulminant hepatitis, "small-forsize" syndrome, acute liver failure on chronic, hepatic insufficiency post-hepatectomy). In these situations, a progressive cholestasis, coagulopathy, encephalopathy, sepsis and multiorgan failure develop; that some authors have related to an insufficient immune response (88-90). Other authors have suggested that an excessive "mitogenic" stimulus would generate the phenomenon known as "hyperproliferative stress response", which induces cellular apoptosis. In necrosis, the passage of intracellular molecules -DAMP- to the extracellular space stimulates the receptors of these patterns (RRP). Among these receptors are the complement system and Toll-like receptors (RTTs) that are expressed in the membranes and cytosol of macrophages, endothelial cells, dendritic cells, NK cells and hepatocytes. DAMPs are also recognized by a subfamily of cytoplasmic receptors of cellular damage and stress, called NLRP and belonging to the NOD-like receptors (NLRs) that once stimulated form the complex called "inflammosome" 
and activate the release of interleukin-1b (IL-b) and interleukin-18 (IL-18), with inflammatory and promitotic effects. The role of the NLRP receptor in inflammation and survival mechanisms has aroused extraordinary interest in recent years (91).

Representation of the initial phase of "purging" the hepatocytes, mediated by the activation of the innate immune system, by PAMP or DAMP (PAMP: patterns Molecules associated with pathogenic microorganisms DAMP: molecular patterns associated with the lesion LPS: lipopolysaccharide $\mathrm{C} 3 \mathrm{a}, \mathrm{C} 5 \mathrm{a}$ : proteolytic fragments of complement proteins C3 and C5 HMGB1: high mobility proteins of box group 1. TLR4: type receptors toll-4 IL6: interleukin 6. TNF: tumor necrosis factor IL-6R: IL-6 receptor JAK: Janus type kinase GP130: IL-6 receptor glycoprotein $130 \mathrm{NF}-\mathrm{K} \beta$ : factor of nuclear transcription Kappa- $\beta$. STAT-3: activator of the transcription and transducer of the signal 3. TNF-R: tumor necrosis factor receptors). Bacteria Cell Necrosis Hepatocyte C. Kupffer C3a C5a Damp's Extracellular space C3a C5a HMGB1 Alarms H2 O TLR4 TNF TNFR IL-6 IL-6R TLR4 PAMP's LPS NF-KB IL-6 TNF NF-KB JAK GP130 Early response genes immediate Reactants acute phase Antiapoptosis Proliferation STAT3 STAT3 STAT3 P STAT3 P (92-95).

It is significant that the recognition proteins of the innate system are phylogenetically prior to the separation between animals and plants (a billion years ago) and to the acquisition of the adaptive immune system. The binding of Toll-like receptors with their ligands stimulates several signaling cascades that culminate in the expression of IL-6, TNF-a and IL-b, and the activation of cytosolic transcription factors-NF-kb, STAT-3, AP-1-that translocate to the nucleus and exert proliferative effects. RTT participate in regeneration by means of pro-survival signals and inhibition of apoptosis in the intestinal mucosa in the colon, lung, skin and in liver regeneration after hepatectomy. HR has also been associated with endogenous ligands present in the enterohepatic circulation: bile acids (Abs), xenobiotics, LPS and with components of the extracellular matrix-fibronectin, heparan sulfate, fibrinogen, hyaluronic acid oligosaccharides- tissue injury (surgical maneuvers) and different types of stress $(93,94)$.

The mesenchymal cells, especially the macrophages, once activated by the DAMP, synthesize proinflammatory cytokines such as IL-1b; TNF-a, IL-6, interferon-gamma (IFN-g), prostaglandins and platelet activating factor (PAF) with proliferative and antiapoptotic functions. The
TNF-a and IL-6 cytokines initiate the preparation phase or "priming" of the hepatocytes (G0-G1 transition of the cell cycle). Said cytokines signal through receptors for TNF (TNF-RI, TNF-RII) and for IL-6 (IL-6R/gp130). They are receptor tyrosine kinases similar to those of growth factors, which activate enzymatic cascades such as mitogen activated protein kinases (MAPK). To this group of kinases belongs the Janus kinase, known as JAK, which once activated phosphorylates the preformed transcription factor STAT-3 (transcriptional activator and signal transducer 3) (JAK/ STAT signaling) (95). The STAT-3 protein binds to DNA promoting the expression of the immediate early response genes (IEGs): c-fos, c-jun and c-myc (called oncogenes), responsible for the start of the cell cycle. More than 180 of these genes have been described that synthesize the proteins necessary to leave the G0 basal state. This phase of preparation or "priming" begins in the first $30 \mathrm{~min}$ and lasts for the first 4 hours posthepatectomy. In addition to the STAT-3 factor, other preformed transcription factors are nuclear factor Kappa-b (NF-kb) and activating protein 1 (AP-1); necessary for the "de novo" synthesis of the regulatory proteins of the transition G0-G1 and G1/S (96).

With genetic manipulation techniques in knock-out and knock-down animals, the participation of STAT-3, NF-kb and AP-1 in liver regeneration has been confirmed. Animals with mutations in TNF-a receptors (TNF-R1), expressed an inhibition of transcription of NF-kb and a severe alteration of regeneration, confirming that transduction of NF-kb in Kupffer cells is crucial in the response to cytokines. KO mice for the gp130 receptors of IL-6 showed minor defects in cell proliferation. In animals KO for IL-6 $\left(\mathrm{IL} 6^{--}\right.$) and its receptor (gp130), the administration of LPS after a hepatectomy reduced survival, demonstrating the protective and stimulatory role of IL-6 in regeneration. The hepato-specific deletion of STAT-3 and AP-1 decreases the expression of cyclins, crucial for the transition of G0-G1 and G1-S of the cell cycle. In contrast, in animals in which an increase in NF-kb factor was induced-through the blockade of its cytoplasmic inhibitor IkbKb-a more intense inflammatory and proliferative response was observed (97).

In addition to the inflammatory mediators-TNF-a, IL-6, C3a, C3b, LPS-, growth factors such as HGF, PDGF and EGF also stimulate the G0-G1 step. HGF signals through its c-met receptor, activates STAT-3 and induces the expression of genes for immediate early response. Mice with a conditional mutation in the Met receptor, express a defect in regeneration. As we will see in the proliferation phase, HGF also regulates the expression of genes related to 
other phases of the cell cycle and has "pro-survival" effects through the inhibition of apoptosis. Complement system and liver regeneration In murine models of liver damage -Cl4C, partial hepatectomy- and in patients undergoing hepatectomy, an increase in the activated fractions of complement $\mathrm{C} 3 \mathrm{a}$ and $\mathrm{C} 5 \mathrm{a}$ has been described in the first 24 hours. In mice deficient $(\mathrm{KO})$ in the $\mathrm{C} 3$ fraction $\left(\mathrm{C}^{-/-}\right)$ and in the $\mathrm{C} 5$ fraction $\left(\mathrm{C}^{-/-}\right)$, alteration in regeneration was observed after a hepatectomy of $70 \%$. In these animals, a reduction in the levels of TNF- $\alpha$ and IL- 6 mRNA was observed, and a decrease in the transcription factors NF$\mathrm{kb}$ and STAT-3. The administration of a $\mathrm{C} 5$ a receptor antagonist (C5a R) had similar effects with an increase in mortality; confirming the role of the complement in the initial phase of priming of hepatocytes a form of response to tissue damage, developed more severe liver lesions than control animals after damage induced by $\mathrm{Cl} 4 \mathrm{C}$ infusion and after a partial hepatectomy $(98,99)$.

In these animals, defects in regeneration and hepatocellular damage reverted after reconstitution with $\mathrm{C} 3 \mathrm{a}$ and $\mathrm{C} 5 \mathrm{a}$. The same and more intense phenomenon was observed in animals with the double mutation $\left(\mathrm{C} 3 / \mathrm{C}^{-/-}\right)$as well as the recovery of regeneration with the administration of $\mathrm{C} 3$ and C5. The repair and trophic functions of the complement system have aroused great interest in tissue regeneration. The complement system, in addition to its action against bacteria, viruses, fungi and tumor cells, is the fastest humoral component to recognize tissue damage through DAMP and initiate the tissue repair response. Platelets and liver regeneration In addition to hemostatic functions, platelets express Toll-like receptors 2, 4 and 9 and participate in the innate immune response $(99,100)$.

These recipients recognize PAMPs and DAMPs, which is why some authors consider them as the "circulating guardians" of tissue damage and a vestige of invertebrate hemocytes. Platelets contain, in their granules, fibrinogen, Von Willebrand factor, adhesion proteins, proangiogenic factors with mitogenic effects, hepatoprotectors (VEGF, PDGF, HGF, IGF, EGF-1 and TGFb), and $95 \%$ of circulating serotonin. Although they are anucleated cells, they have RNA and can synthesize more than 300 different proteins, among which TGFb stands out (101).

The binding of a cytokine with its receptor stimulates the degradation of the inhibitor IkbKb, releasing the factor NFkb that translocates to the nucleus and activates the transcription of the proteins of the acute phase and the aforementioned pro-proliferative and antiapoptotic genes. After a hepatectomy (70\%) or liver damage-ischemia, Cl4C, administration of LPS-a rapid elevation of TNFa and IL-6 occurs. KO mice for IL-6 (IL- $6^{-/-}$) after a hepatectomy developed a hepatic failure that reversed when previously administered IL-6. In animals IL- $6^{-/-}$activation of STAT3 was hardly induced. In conditional KO models for STAT3 (since STAT- ${ }^{-1-}$ is lethal in the embryonic period), its protective effect has been confirmed in adenovirus-induced lesions. In murine models of hepatotoxicity, ischemiareperfusion and severe cholestasis, the cytoprotective effect of IL-6 has been described. The antiapoptotic effect of IL-6 in extreme hepatectomies (87\%) and the rapid regeneration of "small" grafts $(\leq 30 \%)$ has been recently confirmed, avoiding the "small for size" syndrome $(102,103)$. The transcription factor AP-1 promotes the expression of genes of immediate early response in the first 5 hours post-hepatectomy; especially the Jun oncoprotein that facilitates the transition G0-G1 and G1-S. One of the cytokines of the IL-6 family that has aroused great interest is cardiotrophin-1 (CT-1).

The expression of genes related to metabolism is initially supplanted by genes related to cytoskeletal formation, mitotic spindle assembly and mitosis. Transcriptional studies have shown that in the first hours there is a silencing of metabolic genes (first 40 hours after hepatectomy) and a recovery at the end of mitosis. It has been confirmed that the transcription mechanisms dependent on the metabolism of bile acids (Abs), the detoxification of drugs and the regulation of hydrocarbon metabolism, participate in the initial phase of preparation or "priming". Liver regulation of the pool of bile acids in the enterohepatic circulation is well known. In humans, this pool remains between 2 and $4 \mathrm{~g}$, and recirculates about 12 times a day, and in mice it remains at $4 \mathrm{mg}$. An increase in bile acids damages cell membranes causes mitochondrial damage and can lead to apoptosis and cell necrosis. The homeostasis of bile acids is due to nuclear receptors, among which is the "Farnesoid X-Receptor" (FXR) (102-104).

The binding of free and conjugated bile acids with the binding domain of FXR stimulates the transcription of factors involved in the early phases of regeneration (G1-S), such as FoxM1b, and of proliferative genes (Cdc25) $[166,180]$. By performing a hepatectomy of $70 \%$, an abrupt increase in the portal flow to the remaining liver is produced ( $1 / 3$ of the original mass), so the contribution of nutrients and bile acids coming from the splanchnic circulation is tripled. Mice deficient in the FXR receptor show a delay in liver regeneration and an increase in mortality after partial hepatectomy. These animals are unable to activate 
the immediate response genes c-myc, c-fos and c-jun. In contrast, in mice subjected to a partial hepatectomy, the administration of bile acids stimulated the RH, and the administration of cholestyramine delayed said process. Bile acids stimulate the secretion of proinflammatory cytokines such as TNF-a and IL-1b by Kupffer cells, with the effects of prosurvival, antiapoptotic and proliferative $(103,104)$.

Therefore, bile acids and xenobiotics could behave like DANGER molecules stimulating the start of the preparation phase, in addition to the mitogenic effects already discussed on hepatocytes, especially those of a more hydrophobic nature. The relationship of the metabolic pathways with regeneration is complex, since the metabolic variations described can be an epiphenomenon secondary to the early blockage of the "metabolic" genes, without representing a regenerative stimulus per se.

The capacity of the liver to respond to endogenous stimuli - bile acids - and exogenous - xenobiotics -, apart from the mitogenic stimuli of cytokines and growth factors, is unique to the liver and can explain its almost unlimited potential for a "robust" response to stimuli as diverse as a hepatectomy, ischemia or an overload of bile acids (105).

The phase of progression comprises from the G1 phase to cell division. In this period, in addition to chromatin, the cytoplasmic organelles-mitochondria, Golgi apparatus, lysosomes, endoplasmic reticulum-necessary to preserve liver function and maintain a constant relationship between the size of the cytoplasm and the nucleus must be duplicated. The progression phase is regulated by the aforementioned growth factors, HGF, EGF, VEGF and TGF-a, which in addition to mitogenic effects, have trophic and pro-survival effects (antiapoptotic). The primary function of growth factors is to activate the cyclinCdks complexes that initiate and promote changes in the cell cycle: duplication of the mitotic spindle and DNA replication in the $S$ phase. Similar to cytokines, the majority of Growth factors also transmit the mitogenic signal to the nucleus through membrane and cytoplasmic receptors with tyrosine kinase activity (RTK) that promote the cascade of kinases $(104,105)$.

The deficit in regeneration is associated with an increase in cell death and an accumulation of lipids in hepatocytes. At the molecular level an increase in the inhibitory protein of the G1-S transition is observed. Factor FosM1b promotes the expression of late response genes (on the second day post-hepatectomy) and the G2-M transition. Studies in hepato-specific KO mice for FosM1b have confirmed the role of this factor for entry into mitosis and chromosome segregation. It has been observed that hepatocytes overexpressing FosM1b "reconstituted" previously damaged mouse livers, more efficiently than hepatocytes from control animals. This effect was confirmed even in "elderly" hepatocytes, harboring the therapeutic possibility in elderly patients with chronic liver disease (105).

Growth factors and hormones such as insulin that signal through the cascade of Ras-MAP kinases phosphorylate and inhibit GSK3b, allowing the synthesis of glycogen and the activation of pre-formed factors AP-1 and Myc that stimulate cell proliferation.

Due to its mitogenic effect in vivo and in vitro, it is the growth factor that has aroused most interest. In addition to the mitogenic effects it has motógenos, trophic, antiapoptóticos, angiogenic and morphogenic effects in the development of the liver, brain, placenta, lungs, intestine, myocardium and reproductive system. HGF is synthesized by mesenchymal cells and stored as a pro-HGF precursor form in the extracellular matrix (ME). HGF is a glycoprotein very similar to coagulation factors and fibrinolysis (plasminogen). The activation of pro-HGF is due to the proteolytic effect of urokinase-type plasminogen activator (m-PA). After an acute liver injury there is an abrupt increase-10 to 20 times-of plasma HGF. This elevation is due to the release of HGF stored in the EM and the synthesis of HGF by macrophages stimulated by IL- 6 and TNF-a. In addition, HGF is synthesized by mesenchymal cells of other organs-lung, kidney, spleenwhich confirms its endocrine effect (106).

HGF signals through a receptor tyrosine kinase C-met- whose phosphorylation is observed between 1 and 15 minutes after the hepatectomy, up to $60 \mathrm{~min}$. In humans, a more prolonged elevation of HGF has been described; up to two weeks post-hepatectomy. The highest levels have been described in fulminant hepatitis, a finding that questioned its possible therapeutic efficacy in this situation. This paradoxical phenomenon-inability to stimulate the proliferation of hepatocytes and prevent their cell death, with very high levels of HGF- has been attributed to the "blockade of the C-met receptor" in the face of the simultaneous increase -competitive inhibitionof other signaling molecules such as IL-6 and TGF-b1 in the plasma, or to the phenomenon already described as "hyperproliferative stress response", whereby an excessive mitogenic stimulus causes cellular apoptosis by activating p53 (102-104).

The systemic and intraportal infusion of HGF in rodents increases the synthesis of DNA in the hepatocytes of zone 1 , 
and the intraportal infusion of "human" HGF in mice, causes the proliferation of hepatocytes and hepatomegaly. The transfection of the human gene-HGF-by hydrodynamic injection of the DNA plasmid in the mouse induces hepatomegaly by the activation of the b-catenin pathway; and the infusion of high amounts of HGF increases the size of the liver, associated with the stimulation of the mitogenesis. The withdrawal of HGF generated a marked apoptosis and a restoration of the hepatic DNA to the basal levels. The "pretreatment" with collagenase in rats that subsequently received HGF, potentiated the effect of HGF. Likewise, it was observed that hepatocytes isolated by digestion of the parenchyma with collagenase, expressed early markers of cell cycle initiation.

It has been suggested that remodeling of the extracellular matrix - release of metalloproteinases - would be an initial phase of regeneration, sensitizing hepatocytes to HGF stored in the liver. Probably remodeling of the extracellular matrix, mediated by urokinase and metalloproteinases, generate the release of DAMP-derived from tissue damage - capable of generating a sterile inflammatory response through the activation of Toll-like receptors and consequently the activation of the factors of transcription $\mathrm{NF}-\mathrm{Kb}, \mathrm{AP}-1$ and STAT- 3 related to the beginning of the cell cycle. The membrane receptor with tyrosine kinase activity "C-met" is present in epithelial and mesenchymal cells. Activation downstream of the C-met receptor promotes the cascade of mitogen-activated kinases (MAP kinases) and this stimulates cytosolic transcription factors (AP-1) related to proliferation and cell survival. In addition, HGF signals through the Janus-like kinase that activates the transcription factors STAT-3, the nuclear factor NF-Kb and b-catenin (105).

The binding of HGF with the C-met receptor phosphorylates b-catenin, facilitates its translocation to the nucleus and the expression of cyclin D necessary for the G0-G1 transition of the cell cycle. In transgenic mouse models, the importance of HGF and its C-met receptor in the regenerative response of the liver has been confirmed. Deficient mice in HGF and in their C-met receptor, die during pregnancy and express a decrease in hepatic and parenchymal cell size.

The TFG-a is synthesized by the hepatocytes themselves - an autocrine effect-and has paracrine effects on the endothelial cells and the biliary epithelium. After a hepatectomy, the levels of TGF-a increase between 24 and 48 hours. It has been observed that in vitro and in vivo stimulates the synthesis of DNA and, that the addition of
TFG-a in cultures of hepatocytes, stimulates the transition through the control point "R". However, homozygous mice with a deletion in the TFG-a gene express normal regeneration, indicating that TFG-a is "dispensable" in regeneration, reinforcing its "robust" character. In a murine model, with overexpression of human TFG-a, an increase in proliferation and hepatomegaly was observed (103-105).

The regeneration ends with the restoration of the initial hepatic mass necessary to perform the hepatic functions (hepatic index $\sim 2.5 \%$ ). The strict regulation of this index is one of the most surprising properties since it represents a strict control of the cessation of the cell cycle and a remodeling of the "neoformed" tissue. The cessation of regeneration has been related to anti-inflammatory cytokines, proapoptotic cytokines and "hepatostatic" factors such as IL-10, suppressor cytokine signaling proteins (SOCS-3), plasminogen activator inhibitor PAI-1 and especially TGF-b. Studies with microarrays in rodents and in pigs have confirmed that the initial overexpression of pro-mitotic genes is followed by supplanting by metabolic genes and later by those related to the cessation of proliferation and the return to the previous quiescent stage. TGF-b comprises a family of cytokines (TGFb1-3) related to development and healing.

They signal through two receptors (types I and II) that activate the transcription regulators, known as SMAD proteins (Small Mothers Against Decapentaplegic), which are translocated to the nucleus. In most tissues, TGF-b inhibits proliferation in the G1 phase of the cycle. It has been described that after a hepatectomy, mRNA of TGF-b increases early, although there is a transient resistance of hepatocytes to TGF-b due to a decrease in the expression of its receptors and an overexpression of its inhibitors (105). This resistance subsides after the synthesis of DNA, recovering the sensitivity to TGF-b that inhibits cell proliferation and induces the cessation of regeneration (in the rat at 5-7 days, and three weeks in the pig).

In hepatocytes in culture, and in "bioartificial" models, the role of the extracellular matrix in the differentiation and proliferation of hepatocytes has been highlighted. Despite being $0.5 \%$ of the liver weight, the ME plays an active role in the response to tissue damage and regeneration. The ME, in addition to harboring growth factors -PDGF, TGF-b, VEGF, HGF-, contains cytokines, metalloproteinases -collagenase, gelatinase- and macromolecules such as fibronectin, laminin and collagen (types I, III and VI), very reactive when the endothelium-sinusoidal barrier is altered. After a liver injury—surgical trauma, ischemia, toxic, etc.- 
the urokinase-type plasminogen activator (m-PA) and the metalloproteinases are released, which release the growth factors of their precursor forms (pro-HGF $\rightarrow$ HGF) and they degrade macromolecules (fibrinogen, fibronectin, laminin) with the release of molecules -DAMP- that induce the sterile inflammatory response (DANGER theory) and initiate the purging phase of hepatocytes $(106,107)$.

It has been described that the initial proliferation after a hepatectomy is associated with a degradation of the extracellular matrix; leading to accumulations of neoformed hepatocytes, with limited access to endocrine and paracrine factors that, in basal conditions, inhibit cell division. Hypertrophic accumulations require subsequent tissue remodeling until the initial hepatic index is reached. At 14 days after a hepatectomy, the liver of mice deficient in ILK reaches a weight greater than $58 \%$ of the initial weight. In these mice an increase in the expression of HGF and its C-met receptor was observed, as well as a decrease in the cell cycle inhibitor p27 $(106,107)$.

Due to the great interest in the development of "bioartificial livers" through the "reconstitution" of livers matrices previously decellularized with hepatocytes and/ or pluripotent cells, their development has been driven by the growing demand of grafts for transplantation both in the liver, as kidney, lung and heart. p53 and control of regeneration In multicellular organisms, cell cycle control mechanisms exist in situations of stress-lack of nutrients, hypoxia, DNA damage-that arrest the cycle or induce cellular apoptosis.

The most common system is the $\mathrm{p} 53$ protein family, known as "the guardian of the genome" since it responds to DNA damage and other stress situations. Although p53 is known as an "oncosuppressant" factor (have reported more than 25,000 mutations of p53 in human tumors), has recently aroused interest for its role in the control and cessation of RH. The absence of $\mathrm{p} 53$ ( $\mathrm{p} 53^{-/-}$mice) was associated with a much higher degree of ploidy ( $8 \mathrm{n}$ and 16n) due to failures in the final phase of the cycle or in cytokinesis. p53 regulates the expression of the genes involved in the three phases of cell division, startprogression, division and return to the G0 state after hepatectomy.

It is well known that the activation of $\mathrm{p} 53$ stops the cell cycle and induces apoptosis in situations of cellular stress, such as severe hypoxia, acidosis or an excessive mitogenic stimulus. The increase in $\mathrm{p} 53$ activity increases the expression of the p21 inhibitor of the cell cycle and of proapoptotic proteins (caspases), causing a permanent stop of the cell cycle and even cell death. The cellular response to hyperproliferative stress is the in vivo correlate of the one described in vitro as replicative senescence. The cells in culture, after several divisions, suffer the stable arrest of the cell cycle-replicative senescence-due to the increase of $\mathrm{p} 53$. Senescence has been attributed to hyperproliferative stress-Myc and hyperactive Ras-or to in vitro physiological conditions: absence of extracellular matrix components or inadequate oxygen levels (106).

In contrast, cells lacking p53 proliferate indefinitely in cultures and have been termed "immortal". Although the response to hyperproliferative stress has been related to protection mechanisms against cancer, this physiological response could occur in situations with an excessive mitogenic stimulus such as "small liver syndrome", fulminant hepatic failure, hepatic insufficiency posthepatectomy or Hepatic insufficiency over chronic. In these situations, a great mitogenic stimulus is produced derived from endothelial damage- - friction stress", necrosis, parenchymal hemorrhage, arterial vasospasm and hypoperfusion (104-107).

Today it is unknown why in some cases the liver regenerates, and in others, it fails, developing an irreversible liver failure; and there is also no clear criterion about the minimum amount of remaining liver required for hepatic resection or intervenous transplantation. Despite the multitude of works on liver regeneration, its regulatory mechanisms continue to be a mystery.

HR and Fibrosis both are the most enigmatic and extraordinary biological phenomena of adaptation and response to maintain internal homeostasis. In recent decades it has been the subject of intense research due to its therapeutic implications. It is a very complex and strictly regulated phenomenon that obeys the pattern of response to tissue damage, with an initial phase of preparation or "priming" that corresponds to the G0-G1 transition of the cell cycle of the hepatocytes, and a subsequent phase of proliferation-phases S and $M$ - that ends with the restoration of the hepatic mass. This process has been related to hormones (insulin/glucagon), cytokines (TNF-a, IL-6, IL-1b, IL-10), growth factors (HGF, EGF, VEGF) and, in the last decade, mesenchymal hematopoietic stem cells (CD133+, CD) and oval cells (105-108).

The recent generation of structures with "hepatoid" phenotype in rodents from induced pluripotent stem cells (iPS) has generated great expectations as a possible alternative to liver transplantation; although due to the extraordinary complexity of the liver they must be viewed 
with caution. In the $\mathrm{RH}$, the characteristics of the most regulated biological systems (robustness) - pleiomorphism, redundancy and feedback mechanism-are met rigorously, such as the cell cycle, the natural immune response or the circadian rhythm, and that explain the difficulty of its manipulation with therapeutic. Due to the complexity of the liver, attempts to replace its functions temporarily and definitively have been unsuccessful, except for orthotopic liver transplantation. Perhaps the development of cell therapy and knowledge of the molecular and physiological basis of regeneration, can achieve the desired dream of using the regenerative capacity of the liver in the treatment of liver diseases, whose only current alternative is liver transplantation $(108,109)$.

\section{Acknowledgments}

Funding: None.

\section{Footnote}

Conflicts of Interest: Both authors have completed the ICMJE uniform disclosure form (available at https://tgh.amegroups. com/article/view/10.21037/tgh.2020.02.21/coif). The authors have no conflicts of interest to declare.

Ethical Statement: The authors are accountable for all aspects of the work in ensuring that questions related to the accuracy or integrity of any part of the work are appropriately investigated and resolved.

Open Access Statement: This is an Open Access article distributed in accordance with the Creative Commons Attribution-NonCommercial-NoDerivs 4.0 International License (CC BY-NC-ND 4.0), which permits the noncommercial replication and distribution of the article with the strict proviso that no changes or edits are made and the original work is properly cited (including links to both the formal publication through the relevant DOI and the license). See: https://creativecommons.org/licenses/by-nc-nd/4.0/.

\section{References}

1. Wallace K, Burt AD, Wright MC. Liver fibrosis. Biochem J 2008;411:1-18

2. Bataller R, Brenner DA. Liver fibrosis. J Clin Invest 2005;115:209-18.

3. Younossi ZM, Stepanova M, Afendy M, et al. Changes in the prevalence of the most common causes of chronic liver diseases in the United States from 1988 to 2008. Clin Gastroenterol Hepatol 2011;9:524-30.e1; quiz e60.

4. Bhala N, Angulo P, van der Poorten D, et al. The natural history of nonalcoholic fatty liver disease with advanced fibrosis or cirrhosis: an international collaborative study. Hepatology 2011;54:1208-16.

5. Angulo P. Non-alcoholic fatty liver disease. N Engl J Med 2002;346:1221-31.

6. Blachier M, Lefeu H, Rooadosaviljevic MP, et al. The burden of liver disease in Europe. A review of available epidemiological data. J Hepatol 2013;58:593-608.

7. Bravo AA, Sheth SG, Chopra S, et al. Liver biopsy. N Engl J Med 2001;344:495-500.

8. Regev A, Berho M, Jeffers LJ. Sampling error and intra observer variation in liver biopsy in patients with chronic HCV infection. Am J Gastroenterol 2002;97:2614-8.

9. Poynard T, Lenaour G, Vaillant JC, et al. Liver biopsy analysis has a low level of performance for diagnosis of intermediate stages of fibrosis. Clin Gastroenterol Hepatol 2012;10:657-63.e7.

10. Forbes SJ, Parola M. Liver fibrogenic cells. Best Pract Res Clin Gastroenterol 2011;25:207-17.

11. Debbaut C, Segers P, Cornillie P, et al. Analyzing the human liver vascular architecture by combining vascular corrosion casting and micro-CT scanning: a feasibility study. J Anat 2014;224:509-17.

12. Pellicoro A, Ramachandran P, Iredale JP, et al. Liver fibrosis and repair: immune regulation of wound healing in a solid organ. Nat Rev Immunol 2014;14:181-94.

13. Aghemo A, Colombo M. Cirrhosis regression in chronic hepatitis $\mathrm{C}$ : an old tale with a new ending. Gastroenterology 2009;136:1447-49.

14. Friedman SL. Mechanisms of Hepatic fibrogenesis. Gastroenterology 2008;134:1655-69.

15. Holt AP, Salmon M, Buckley CD, et al. Immune interactions in hepatic fibrosis. Clin Liver Dis 2008;12:861-82.

16. Juárez H, Figueroa JL, Hernández E, et al. Lesiones anatomopatológicas hepáticas por la obstrucción de la vía biliar extrahepática en ratones 2008. Available online: http://www.revistagastroenterologiamexico.org/eslesiones-anatomopatologicas-hepaticas-por-obstruccionarticulo-13131666

17. Li Y, Chang M, Abar O, et al. Multiple variants in tolllike receptor 4 gene modulate risk of liver fibrosis in Caucasians with chronic hepatitis $\mathrm{C}$ infection. J Hepatol 2009;51:750-7. 
18. Peeters G, Debbaut C, Friebel A, et al. Quantitative analysis of hepatic macro- and microvascular alterations during cirrhogenesis in the rat. J Anat 2018;232:485-96.

19. Li X, Benjamin IS, Alexander B. Reproducible production of thioacetamide-induced macronodular cirrhosis in the rat with no mortality. J Hepatol 2002;36:488-93.

20. Anthony PP, Ishak KG, Nayak NC, et al. The morphology of cirrhosis. Recommendations on definition, nomenclature, and classification by a working group sponsored by the World Health Organization. J Clin Pathol 1978;31:395-414.

21. García-Tsao G, Friedman S, Iredale J, et al. Now there are many (stages) where before there was one: In search of a pathophysiological classification of cirrhosis Hepatology 2010;51:1445-9.

22. Desmet VJ, Roskams T. Cirrhosis reversal: a duel between dogma and myth. J Hepatol 2004;40:860-7.

23. Friedman SL, Bansal MB. Reversal of hepatic fibrosis-fact or fantasy? Hepatology 2006;43:S82-8.

24. D'Amico G, Morabito A, Pagliaro L, et al. Survival and prognostic indicators in compensated and decompensated cirrhosis. Dig Dis Sci 1986;31:468-75.

25. A. Hatzakis A, S. Wait S, J. Bruix J, et al. The state of hepatitis B and $\mathrm{C}$ in Europe: report from the hepatitis $\mathrm{B}$ and C summit conference. J Viral Hepat 2011;18:1-16.

26. Kanwal F, Hoang T, Kramer JR, et al. Increasing prevalence of HCC and cirrhosis in patients with chronic hepatitis $\mathrm{C}$ virus infection. Gastroenterology 2011;140:1182-8.e1.

27. European Association For The Study Of The Liver. EASL Clinical Practice Guidelines: management of chronic hepatitis B. J Hepatol 2009;50:227-42.

28. Fattovich G, Bortolotti F, Donato F. Natural history of chronic hepatitis B: special emphasis on disease progression and prognostic factors. J Hepatol 2008;48:335-52.

29. Weisberg IS, Brown RS, Sigal SH. Hepatitis B and endstage liver disease. Clin Liver Dis 2007;11:893-916.

30. O'Shea RS, Dasarathy S, McCullough AJ. Practice Guideline Committee of the American Association for the Study of Liver Diseases. Practice Parameters Committee of the American College of Gastroenterology. Alcoholic liver disease. Hepatology 2010;51:307-28.

31. Sanyal AJ, Bonos C, Sargeant C, et al. Similarities and differences in outcomes of cirrhosis due to nonalcoholic steatohepatitis and hepatitis C. Hepatology 2006;43:682-9.

32. Manns MP, Czaja AJ, Gorham JD, et al. American Association for the Study of Liver Diseases. Diagnosis and management of autoimmune hepatitis Hepatology 2010;51:2193-213.

33. Lindor KD, Gershwin ME, Poupon R, et al. Primary biliary cirrhosis. Hepatology 2009;50:291-308.

34. DeLeve LD, Valla DC, Garcia-Tsao G, et al. Vascular disorders of the liver. Hepatology 2009;49:1729-64.

35. Bacon BR, Adams PC, Kowdley KV, et al. Diagnosis and management of hemochromatosis: 2011 practice guideline by the American Association for the Study of Liver Diseases. Hepatology 2011;54:328-43.

36. Roberts EA, Schilsky ML, American Association for Study of Liver Diseases (AASLD). Diagnosis and treatment of Wilson disease: an update. Hepatology 2008;47:2089-111.

37. Lee UE, Friedman SL. Mechanisms of hepatic fibrogénesis. Best Pract Res Clin Gastroenterol 2011;25:195-206.

38. Benvegnù L, Gios M, Boccato S, et al. Natural history of compensa viral cirrhosis: a prospective study on the incidence and hierarchy of major complications. Gut 2004;53:744-9.

39. D’Amico G, García-Tsao G, Pagliaro L. Natural history and prognostic indicators of survival in cirrhosis: a systematic review of 118 studies. J Hepatol 2006;44:217-31.

40. Runyon BA. AASLD Practice Guidelines Committee. Management of adult patients with ascites due to cirrhosis: an update Hepatology 2009;49:2087-107.

41. Nagula S, Jain D, Groszmann RJ, et al. Histologicalhemody correlation in cirrhosis-a histological classification of the severity of cirrhosis. J Hepatol 2006;44:111-7.

42. de Franchis R, Baveno V. Faculty. Revising consensus in portal hypertension: report of the Baveno $\mathrm{V}$ consensus workshop on methodology of diagnosis and therapy in portal hypertension. J Hepatol 2010;53:762-8.

43. Child CG, Turcotte JG. Surgery and portal hypertension. Major Probl Clin Surg 1964;1:1-85.

44. Pugh RN, Murray-Lyon IM, Dawson JL, et al. Transection of the oesophagus for bleeding oesophageal varices. Br J Surg 1973;60:646-9.

45. Kamath PS, Wiesner RH, Malinchoc M, et al. A model to predict survival in patients with end-stage liver disease. Hepatology 2001;33:464-70.

46. Wiesner R, Edwards E, Freeman R, et al. United Network for Organ Sharing Liver Disease Severity Score Committee. Model for end-stage liver disease (MELD) and allocation of donor livers. Gastroenterology 2003;124:91-6.

47. Durand F, Valla D. Assessment of the prognosis of cirrhosis: Child-Pugh versus MELD. J Hepatol 
2005;42:S100-7.

48. Suman A, Barnes DS, Zein NN, et al. Predicting outcome after cardiac surgery in patients with cirrhosis: a comparison of Child-Pugh and MELD scores. Clin Gastroenterol Hepatol 2004;2:719-23.

49. Baffy G. Kupffer cells in non-alcoholic fatty liver disease: The emerging view. J Hepatol 2009;51:212-23.

50. Michalopoulos GK. Liver regeneration after partial hepatectomy critical analysis of mechanistic. Am J Pathol 2010;176:2-13.

51. Zou Y, Bao Q, Kumar S, et al. Four waves of hepatocyte proliferation linked with three waves of hepatic fat accumulation during partial hepatectomy-induced liver regeneration. PLoS One 2012;7:e30675.

52. Rudnick DA, Davidson NO. Functional relationships between lipidmetabolism and liver regeneration. Int J Hepatol 2012;2012:549241.

53. Gazit V, Weymann A, Hartman E, et al. Liver Regeneration is Impaired in Lipodystrophic fld Mice. Hepatology 2010;52:2109-17.

54. Sanal MG. The blind men 'see' the elephant-the many faces of fatty liver disease. World J Gastroenterol 2008;14:831-44.

55. Veteläinen R, van Vliet A, Gouma DJ, et al. Steatosis as a Risk Factor in Liver Surgery. Ann Surg 2007;245:20-30.

56. Dowman JK, Tomlinson JW, Newsome PN. Pathogenesis of non-alcoholic fatty liver disease. QJM 2010;103:71-83.

57. Rensen SS, Slaats Y, Driessen A, et al. Activation of the Complement System in Human Nonalcoholic Fatty Liver Disease. Hepatology 2009;50:1809-17.

58. Braunersreuther V, Viviani GL, Mach F, et al. Role of cytokines and chemokines in non-alcoholic fatty liver disease. World J Gastroenterol 2012;18:727-35.

59. Jarrar MH, Baranova A, Collantes R, et al. Adipokines and cytokines in non-alcoholic fatty liver disease. Aliment Pharmacol Ther 2008;27:412-21.

60. Hatziagelaki E, Karageorgopoulos DE, Chounta A, et al. Predictors of impaired glucose regulation in patients with non-alcoholic fatty liver disease. Exp Diabetes Res 2012;2012:351974.

61. Veteläinen R, van Vliet AK, van Gulik TM. Severe steatosis increases hepatocellular injury and impairs liver regeneration in a rat model of partial hepatectomy. Ann Surg 2007;245:44-50.

62. Newberry EP, Kennedy SM, Xie Y, et al. Altered Hepatic Triglyceride Content After Partial Hepatectomy Without Impaired Liver Regeneration in Multiple Murine Genetic
Models. Hepatology 2008;48:1097-105.

63. Ben Mosbah I, Alfany-Fernández I, Martel C, et al. Endoplasmic reticulum stress inhibition protects steatotic and non-steatotic livers in partial hepatectomy under ischemia-reperfusion. Cell Death Dis 2010 8;1:e52.

64. Damrauer SM, Studer P, da Silva CG, et al. A20 Modulates Lipid Metabolism and Energy Production to Promote Liver Regeneration. PLoS One 2011;6:e17715.

65. Broering R, Lu M, Schlaak JF. Role of Toll-like receptors in liver health and disease. Clin Sci (Lond) 2011;121:415-26.

66. Rivera CA, Adegboyega P, van Rooijen N, et al. Toll-like receptor-4 signaling and Kupffer cells play pivotal roles in the pathogenesis of non-alcoholic steatohepatitis. J Hepatol 2007;47:571-9.

67. Bertola A, Bonnafous S, Anty R, et al. Hepatic Expression Patterns of Inflammatory and Immune Response Genes Associated with Obesity and NASH in Morbidly Obese Patients. PLoS One 2010;5:e13577.

68. Matsumoto K, Yu S, Jia Y, et al. Critical role for transcription coactivator peroxisome proliferator-activated receptor (PPAR)-binding protein/TRAP220 in liver regeneration and PPARalpha ligand-induced liver tumor development. J Biol Chem 2007;282:17053-60.

69. Shi Y, Massague J. Mechanisms of TGF-beta signaling from cell membrane to the nucleus. Cell 2003;113:685-700.

70. Moustakas A, Heldin CH. The regulation of TGFbeta signal transduction. Development 2009;136:3699-714.

71. Brown KA, Pietenpol JA, Moses HL. A tale of two proteins: Differential roles and regulation of Smad2 and Smad3 in TGF-beta signaling. J Cell Biochem 2007;101:9-33.

72. Chari RS, Price DT, Sue SR, et al. Down-regulation of transforming growth factor beta receptor type I, II, and III during liver regeneration. Am J Surg 1995;169:126-31; discussion 131-2.

73. Macias-Silva M, Li W, Leu JI, et al. Up-regulated transcriptional repressors SnoN and Ski bind Smad proteins to antagonize transforming growth factorbeta signals during liver regeneration. J Biol Chem 2002;277:28483-90.

74. Nishikawa Y, Wang M, Carr BI. Changes in TGFbeta receptors of rat hepatocytes during primary culture and liver regeneration: increased expression of TGFbeta receptors associated with increased sensitivity to TGF-beta-mediated growth inhibition. J Cell Physiol 1998;176:612-23.

75. Russell WE, Coffey RJ Jr, Ouellette AJ, et al. Type beta 
transforming growth factor reversibly inhibits the early proliferative response to partial hepatectomy in the rat. Proc Natl Acad Sci U S A 1988;85:5126-30.

76. Bouzahzah B, Fu M, Iavarone A, et al. Transforming growth factor-beta1 recruits histone deacetylase 1 to a p130 repressor complex in transgenic mice in vivo. Cancer Res 2000;60:4531-7.

77. Oe S, Lemmer ER, Conner EA, et al. Intact signaling by transforming growth factor beta is not required for termination of liver regeneration in mice. Hepatology 2004;40:1098-105.

78. Kogure K, Zhang YQ, Maeshima A, et al. The role of activin and transforming growth factor-beta in the regulation of organ mass in the rat liver. Hepatology 2000;31:916-21.

79. Uygun BE, Soto-Gutierrez A, Yagi H, et al. Organ reengineering through development of a transplantable recellularized liver graft using decellularized liver matrix. Nat Med 2010;16:814-20.

80. Uygun BE, Yarmush ML, Uygun K. Application of whole-organ tissue engineering in hepatology. Nat Rev Gastroenterol Hepatol 2012;9:738-44.

81. Hammond JS, Gilbert TW, Howard D, et al. Scaffolds containing growth factors and extracellular matrix induce hepatocyte proliferation and cell migration in normal and regenerating rat liver. J Hepatol 2011;54:279-87.

82. Badylak SF, Taylor D, Uygun K. Whole-organ tissue engineering: Decellularization and recellularization of three-dimensional matrix scaffolds. Annu Rev Biomed Eng 2011;13:27-53

83. Baptista PM, Siddiqui MM, Lozier G, et al. The use of whole organ decellularization for the generation of a vascularized liver organoid. Hepatology 2011;53:604-17.

84. Gkretsi V, Bowen WC, Yang Y, et al. Integrinlinked kinase is involved in matrix-induced hepatocyte differentiation. Biochem Biophys Res Commun 2007;353:638-43.

85. Gkretsi V, Apte U, Mars WM, et al. Liver-specific ablation of integrin-linked kinase in mice results in abnormal histology, enhanced cell proliferation, and hepatomegaly. Hepatology 2008;48:1932-41.

86. Apte U, Gkretsi V, Bowen WC, et al. Enhanced liver regeneration following changes induced by hepatocytespecific genetic ablation of integrin-linked kinase. Hepatology 2009;50:844-51.

87. Shafiei MS, Rockey DC. The role of integrin-linked kinase in liver wound healing. J Biol Chem 2006;281:24863-72.

88. Song JJ, Guyette JP, Gilpin SE, et al. Regeneration and experimental orthotopic transplantation of a bioengineered kidney. Nat Med 2013;19:646-51.

89. Song JJ, Kim SS, Liu Z, et al. Enhanced in vivo function of bioartificial lungs in rats. Ann Thorac Surg 2011;92:9981005; discussion 1005-6.

90. Bakkenist CJ, Kastan MB. Initiating cellular stress responses. Cell 2004;118:9-17.

91. Ben-Porath I, Weinberg RA. When cells get stressed: An integrative view of cellular senescence. J Clin Invest 2004;113:8-13.

92. Morgan DO. The DNA damage response. In: Morgan DO. The cell cycle: Principles of control. London: New Science Press, 2007:227-45.

93. Campisi J. Senescent cells, tumor suppression, and organismal aging: Good citizens, bad neighbors. Cell 2005;120:513-22.

94. Weinberg RA. P53 and apoptosis: master guardian and executioner. In: Weinberg RA. The biology of cancer. New York: Garland Science, 2007:307-56.

95. Vousden KH, Lu X. Live or let die: the cell's response to p53. Nat Rev Cancer 2002;2:594-604.

96. Efeyan A, Serrano M. P53: Guardian of the genome and policeman of the oncogenes. Cell Cycle 2007;6:1006-10.

97. Li CC, Chu HY, Yang CW, et al. Aurora-A overexpression in mouse liver causes p53-dependent premitotic arrest during liver regeneration. Mol Cancer Res 2009;7:678-88.

98. Maddocks OD, Vousden KH. Metabolic regulation by p53. J Mol Med (Berl) 2011;89:237-45.

99. Fededa JP, Gerlich DW. Molecular control of animal cell cytokinesis. Nat Cell Biol 2012;14:440-7.

100. Gallagher SJ, Kefford RF, Rizos H. The ARF tumour suppressor. Int J Biochem Cell Biol 2006;38:1637-41.

101. Wadhwa R, Sugihara T, Taira K, et al. The ARF-p53 senescence pathway in mouse and human cells. Histol Histopathol 2004;19:311-6.

102.Zhou J, Schmid T, Schnitzer S, et al. Tumor hypoxia and cancer progression. Cancer Lett 2006;237:10-21.

103. Demetris AJ, Seaberg EC, Wennerberg A, et al. Ductular reaction after submassive necrosis in humans. Special emphasis on analysis of ductular hepatocytes. Am J Pathol 1996;149:439-48.

104. Stravitz RT, Kramer DJ. Acute liver failure. In: Boyer TD, Manns MP, Sanyal AJ. editors. Zakim and Boyer's hepatology: a textbook of liver disease. 6th ed. Philadelphia: Saunders Elsevier, 2012:327-51.

105. Oda M, Yokomori H, Han JY. Regulatory mechanisms of hepatic microcirculatory hemodynamics: hepatic arterial system. Clin Hemorheol Microcirc 2006;34:11-26. 
106. Ninomiya M, Shirabe K, Terashi T, et al. Deceleration of regenerative response improves the outcome of rat with massive hepatectomy. Am J Transplant 2010;10:1580-7.

107. Markiewski MM, DeAngelis RA, Lambris JD. Liver inflammation and regeneration: Two distinct biological phenomena or parallel pathophysiologic processes? Mol
Immunol 2006;43:45-56.

108. Clavien PA, Oberkofler CE, Raptis DA, et al. What is critical for liver surgery and partial liver transplantation: Size or quality? Hepatology 2010;52:715-29.

109. Takebe T, Sekine K, Enomura M, et al. Vascularized and functional human liver from an iPSC-derived organ bud transplant. Nature 2013;499:481-4. doi: $10.21037 / \operatorname{tgh} .2020 .02 .21$

Cite this article as: Zuñiga-Aguilar E, Ramírez-Fernández O. Fibrosis and hepatic regeneration mechanism. Transl Gastroenterol Hepatol 2022;7:9. 